\title{
Compromising system and user interests in shelter location and evacuation planning
}

\author{
Vedat Bayram*, Barbaros Ç. Tansel ${ }^{\dagger}$, Hande Yaman \\ Bilkent University, Department of Industrial Engineering, Bilkent, 06800 Ankara, Turkey
}

\section{A R T I C L E I N F O}

\section{Article history:}

Received 9 May 2014

Received in revised form 23 November 2014

Accepted 24 November 2014

Available online 26 December 2014

\section{Keywords:}

Evacuation traffic management

Shelter location

Traffic assignment

System optimal

Constrained system optimal

Second order cone programming

\begin{abstract}
A B S T R A C T
Traffic management during an evacuation and the decision of where to locate the shelters are of critical importance to the performance of an evacuation plan. From the evacuation management authority's point of view, the desirable goal is to minimize the total evacuation time by computing a system optimum (SO). However, evacuees may not be willing to take long routes enforced on them by a SO solution; but they may consent to taking routes with lengths not longer than the shortest path to the nearest shelter site by more than a tolerable factor. We develop a model that optimally locates shelters and assigns evacuees to the nearest shelter sites by assigning them to shortest paths, shortest and nearest with a given degree of tolerance, so that the total evacuation time is minimized. As the travel time on a road segment is often modeled as a nonlinear function of the flow on the segment, the resulting model is a nonlinear mixed integer programming model. We develop a solution method that can handle practical size problems using second order cone programming techniques. Using our model, we investigate the importance of the number and locations of shelter sites and the trade-off between efficiency and fairness.
\end{abstract}

(C) 2014 Elsevier Ltd. All rights reserved.

\section{Introduction}

There has been a significant increase in the number of disasters over the past decades; from fewer than 50 disasters per year reported in 1950 to more than 400 disasters in 2010 (EM-DAT, 2013). Consequently, the number of people affected and the economic damages caused by disasters increased. International Federation of Red Cross and Red Crescent Societies (IFRC, 2011) defines disasters as "serious disruptions of the functioning of a community through widespread losses that exceed the community's capacity to cope with using its own resources". IFRC classifies disasters as naturally occurring physical phenomena caused either by rapid or slow onset events which can be geophysical, hydrological, climatological, meteorological or biological and as technological or man-made hazards that are caused by humans and occur in or close to human settlements. Federal Emergency Management Agency (FEMA) reports that 45-75 disasters require an evacuation annually (TRB, 2008). Whether it is made by the Military or Civil Emergency Management authorities, evacuation planning for large scale disasters such as earthquakes, hurricanes, floods, tsunamis or CBRN (Chemical, Biological, Radiological and Nuclear) consequences of ballistic missile attacks is of critical importance for disaster management.

Various traffic management problems arise during disasters; evacuation of the disaster region being one of the most important. In 1999 hurricane Floyd (CNN.com, 2001), and in 2005 hurricanes Katrina and Rita (TRB, 2008) required millions

\footnotetext{
* Corresponding author.

E-mail addresses: bayram@bilkent.edu.tr (V. Bayram), hyaman@bilkent.edu.tr (H. Yaman).

+ Deceased.
} 
of people to evacuate creating largest traffic jams in the U.S. history. Since disasters have different peculiarities, the evacuation objectives and decisions faced by a disaster management agency may differ. Most frequently used objectives in evacuation models are minimizing the total or average evacuation time, minimizing the clearance time, minimizing the maximum latency and maximizing the number of people who reach safety in a given time period. Clearance time is the time that the last vehicle in the network leaves the danger zone and reaches safety while latency is defined as the total time it takes a vehicle to complete its trip on a given route. The total evacuation time, i.e., the sum of the evacuation times of all vehicles, which is the focus of this paper, is a measure of how long the vehicles stay in the area at risk.

The time to evacuate a disaster region depends on the locations of shelters and on the traffic assignment. Shelters serve as safe facilities to provide the evacuees with food, accommodation and medical care. But the primary goal of sheltering before or after a disaster hits is to protect the population from possible dangers. Sherali et al. (1991) point out at their study that one of the greatest tasks in developing a hurricane evacuation plan is to determine where evacuees should seek shelter in order to retreat from the storm's damaging power. In their study Liu et al. (1996) emphasize that improving the local warning system will be effective only if people at risk can be evacuated to safe shelters. And secure shelters are a means to increase evacuation rates (Litman, 2006). Even though the decision of where to locate the shelters from among potential alternatives is of critical importance to the performance of an evacuation plan (Sherali et al., 1991; Kongsomsaksakul et al., 2005; Kulshrestha et al., 2011), few evacuation models in the literature decide optimally on the number and location of shelters. The aim of this study is to provide an evacuation planning tool that simultaneously optimizes the shelter locations and the allocations of evacuees to shelters and to routes.

The existing models used for assigning evacuees to routes are mostly based on three traffic assignment models, namely, the user equilibrium (UE, also known as User Optimal or Nash Equilibrium), the system optimal (SO) and the nearest allocation (NA) models. These models differ in assumed driver behaviors. In accordance with the UE principle, travelers' aim is to minimize their individual travel times. While a user equilibrium satisfies the drivers, it does not necessarily minimize the total evacuation time in the system. From the evacuation traffic management authority's point of view, the desirable goal is to explicitly minimize the total evacuation time by computing a system optimum. Under SO conditions some travelers may travel longer than they could to the benefit of the overall system. In the NA model, each evacuee uses a shortest path based on geographical distance or free flow travel time to reach the nearest shelter. Clearly, such a traffic assignment may lead to poor system efficiency.

The UE approach is not realistic to plan an evacuation during a disaster for the following reasons. In the UE model, it is assumed that the evacuees have full information on travel times on every possible route and they are able to find the optimal routes. Disasters and evacuations are rare events with unusual traffic demand resulting in different from normal traffic conditions. As a result, evacuees do not have the opportunity to learn from the past experience which routes minimize their evacuation time (Pel et al., 2012). It is unlikely for an equilibrium that distributes demand evenly across the evacuation routes to emerge (Lindell and Prater, 2007). Galindo and Batta (2013) and Faturechi and Miller-Hooks (2014) also state that the assumption that evacuees have perfect information about the road network and the traffic conditions is unrealistic, since it takes a while to state the traffic conditions. Such knowledge hardly exists for emergency evacuation for which the evacuees will have very limited if any prior experience regarding the travel patterns (Yazıc1, 2010).

On the other hand, the SO model, in which a central authority assigns evacuees to routes to minimize the total evacuation time, may route some evacuees on paths much longer than the ones they could take if they had a choice. In a disaster, where the aim of an evacuee is to leave the endangered zone as soon as possible and to reach safety at a shelter point, people may not show conscientious behavior to accept routes that are much longer than the shortest ones they would take. It is likely that some may not abide by the evacuation rules imposed on them; instead they may choose routes to reach the closest shelter site as quickly as possible without considering the adverse affects of their choice on others.

Barrett et al. (2000) classifies destination choices of evacuees as nearest safe destination, soonest safe destination and easiest safe destination. A similar classification is made by Southworth (1991). In a disaster situation, where there is limited information on the road network and congestion levels, evacuees show selfish behavior, as people do even under normal daily traffic conditions (Roughgarden, 2002; Jahn et al., 2003; Schulz and Moses, 2003; Correa et al., 2005, 2007; Schulz and Stier-Moses, 2006; Olsthoorn, 2012) and they tend to select routes that take them to the nearest shelter site, as proposed and implemented by Yamada (1996), Cova and Johnson (2003), Alçada-Almeida et al. (2009), Coutinho-Rodrigues et al. (2012) and Sheu and Pan (2014).

To develop a route guidance system, Jahn et al. (2005) propose to honor the individual needs by imposing additional constraints to ensure that drivers are assigned to "acceptable" paths only. Such a traffic assignment model is referred to as constrained system optimal (CSO).

Our aim is to propose a CSO model that optimally locates shelter sites and that assigns evacuees to the nearest shelter sites and to shortest paths to those shelter sites, shortest and nearest within a given degree of tolerance. As our model already considers fairness among evacuees by assigning them to close shelter sites, we use the overall system performance in our objective and minimize the total evacuation time. We note here that our model generalizes both SO and NA traffic assignment models, as these correspond to the cases of infinite and zero tolerance levels, respectively. The solution of the model evacuates the disaster region as quickly as possible, with a "fair" assignment of evacuees to shelters and to routes. To this end, we propose a nonlinear mixed integer programming model and solve practical size problems in reasonable times by representing the nonlinear objective function with second order cone programming. In addition, we present a sensitivity analysis by changing the level of tolerance and the number of shelters to open and make a comparison of the results of SO, 
NA and CSO approaches based on system performance and fairness. We measure the efficiency of the system by employing performance measures such as total evacuation time, percentage of evacuees reaching safety up to a specified time $T$, maximum latency and price of fairness. As most evacuation planning models in the literature, and as suggested by FEMA (2010), our model is not specific to a certain type of disaster. The specifics of a disaster are represented in the parameters. Consequently, the model can be used both for pre and post disaster management.

In our experiments, we observe that the SO solution may have unacceptable levels of unfairness whereas the solution in which the evacuees travel to the nearest shelter using a shortest path may result in substantial deterioration in system performance. Even small levels of tolerance on the side of the evacuees improves both the system performance and the unfairness measures significantly. Overall, we can conclude that the location and the number of shelters opened drastically affect the evacuation plan and for a carefully selected number of shelters and tolerance level, an efficient yet fair evacuation plan can be achieved.

The rest of the paper is organized as follows. In Section 2, we review the literature on shelter location and evacuation planning. In Section 3, we define our problem formally, show that it is NP-Hard and give a second order conic mixed integer programming formulation. We compare the results with different traffic assignment models in Section 4 and conclude in Section 5.

\section{Literature review}

Few of the evacuation planning models we have encountered in the literature optimally decide on the number and location of shelters to minimize the total system cost or to maximize the benefit.

Yamada (1996) uses the shortest path (nearest allocation) and minimum cost flow approaches to assign pedestrian evacuees to shelters and to routes. These approaches minimize the total distance traveled and disregard the evacuation traffic congestion. Cova and Johnson (2003) propose to use lane-based routing to reduce the delays at the intersections. They present a network flow model to minimize the total distance traveled. Yazıcı and Özbay (2007) and Chiu et al. (2007) use a cell transmission model (CTM) based SO dynamic traffic assignment (DTA) approach. Ng et al. (2010) present a bi-level model that assigns evacuees to shelters in a SO manner in the upper level, and in the lower level evacuees reach their assigned shelters in a UE manner. Hu et al. (2014) propose a mixed-integer linear programming model that considers a multi-step evacuation and temporary resettlement. The model minimizes panic-induced psychological penalty cost, psychological intervention cost, transportation cost and cost of building shelters. These studies do not consider optimal selection of shelter sites among potential ones. Yazıcı and Özbay (2007) perform sensitivity analysis to find out the appropriate locations of shelter sites and Chiu et al. (2007) consider all the nodes at the boundary between the danger zone and the safe zone but inside the safe zone as potential shelter sites and suggest that a shelter is opened at a node if there is a flow into it at the optimal solution.

The location-allocation models that consider the optimal selection of shelter sites are either single level models with a SO approach or bi-level models that specify the locations of shelter sites in a SO manner at the upper level, while assigning evacuees to shelters and routes in a UE manner at the lower level. Sherali et al. (1991) develop a location-allocation model in which the selection of shelter sites and the assignment of the evacuees to the routes are specified in a SO manner. Kongsomsaksakul et al. (2005) study the impact of the shelter locations on evacuation traffic flow management. At the upper level their model determines the number and locations of the shelter sites with the objective of minimizing the total network evacuation time. The lower level is a static UE formulation and given the number and location of the shelter sites, the evacuees choose their routes and the shelter sites that they travel to. Kulshrestha et al. (2011) develop a robust bi-level model that considers demand uncertainty and minimizes the total cost to establish and operate shelters at the upper level while assigning evacuees to shelters and routes in a UE manner at the lower level. Shen et al. (2008) develop scenario based, stochastic, bi-level models that minimize the maximum UE travel time among all node shelter pairs by locating shelters at the upper level and assigning evacuees to shelters and routes in a UE manner at the lower level. Li et al. (2012) propose a scenario based location model for identifying a set of shelter locations that are robust for a range of hurricane events. Their model is a DTA-based stochastic bi-level programming model in which at the upper level the central authority selects the shelter sites for a particular scenario. The objective of the upper-level problem is to minimize the weighted sum of the expected unmet shelter demand and the expected total network travel time. In the lower level, evacuees choose their routes in a dynamic UE manner. Sheu and Pan (2014) propose a method for designing an integrated emergency supply network that utilizes a three-stage multi-objective programming problem. The first stage of their method designs the shelter network for evacuation with a nearest allocation approach as one of the objectives.

Alçada-Almeida et al. (2009) and Coutinho-Rodrigues et al. (2012) introduce a multi-objective approach to identify the number and location of rescue facilities (shelters) and primary and secondary evacuation routes. Their models can be regarded as a multi-objective extension of the $p$-median model. No congestion effect is included in these models, instead average travel velocity is used.

The location allocation models proposed by Kongsomsaksakul et al. (2005), Shen et al. (2008), Ng et al. (2010), Li et al. (2012) are solved using heuristic algorithms and the ones developed by Alçada-Almeida et al. (2009), Coutinho-Rodrigues et al. (2012) and Sheu and Pan (2014) are solved to optimality by exact solution methodologies. Kulshrestha et al. (2011) employ an approximation based cutting plane algorithm. Hamacher et al. (2011) introduce a model and heuristic algorithms 
using a time expanded network for their problem. Sherali et al. (1991) develop both a heuristic and an exact algorithm to solve their model.

Jahn et al. (2005) propose a SO traffic assignment model that includes user constraints to be fair to drivers. They define unfairness as a measure of the detriment for users as the ratio of the traversal time of the recommended path to that of the shortest possible path the user could have taken. The normal length of a path, is defined as either its free flow travel time, its traversal time in UE, its geographic distance, or any other measure that does not depend on the actual flow on the path. They look for a constrained system optimum in which no path carrying positive flow between a certain origin-destination pair is allowed to exceed the normal length of a shortest path between the same origin-destination pair by more than a tolerable factor. They use a variant of the convex combination algorithm of Frank and Wolfe (1956) combined with column generation method to solve their problem. Jahn et al. (2000, 2003), Schulz and Stier-Moses (2006), Li and Zhao (2008), Zhou and Li (2012) develop models and algorithms that consider user needs while trying to achieve the system optimal to find solutions that are fair and efficient at the same time. These models are developed for traffic management in every day normal traffic conditions and do not consider the location of facilities and allocation of drivers.

A related notion is that of satisficing, advanced by Simon (1955), as a model of bounded rational decision making that seeks an acceptable solution rather than a necessarily optimal one, where acceptability is generally defined in relation to some threshold or aspiration level (Mahmassani and Chang, 1987; Mahmassani and Liu, 1999). Following the notion explained by Mahmassani and Chang (1987) and Chen et al. (1997), Lou et al. (2010) define travelers with bounded rationality as those who always choose routes with no cycle and do not necessarily switch to the shortest routes when the difference between the travel times on their current routes and the shortest one is no larger than a threshold value. Szeto and Lo (2006) call this tolerance based dynamic user optimal principle. To find the bounded rational user equilibrium they formulate and solve mathematical programs with complementarity constraints and propose heuristic algorithms.

In this study, we propose a CSO model that locates the shelter sites in a SO manner and that assigns evacuees to the nearest shelter sites by assigning them to the shortest paths to their nearest shelter sites, shortest and nearest with a given degree of tolerance, so that the total evacuation time is minimized. Our aim is to meet both the system needs and the user interests in an evacuation by finding an efficient solution that evacuates the disaster region as quickly as possible and that is fair to the evacuees. Our contributions are: (1) We introduce a novel model that combines the decision making of location of shelters and allocation of evacuees to shelters and routes. In that sense our model is a location-allocation model and in contrast to most of the location-allocation models in the literature that take into account congestion, ours is a single level model that compromises system and user needs. (2) We reformulate our problem using second order conic constraints and solve real size problems exactly using a commercial solver. (3) We present a sensitivity analysis by changing the level of tolerance and the number of shelters to open and make a comparison of the results of SO, NA and CSO approaches based on system performance and fairness. (4) We analyze the impact of having capacitated shelters on performance measures.

\section{Models}

\subsection{Travel time function}

Generally, travel times are considered to be positive and monotonically increasing functions of traffic flow, since an increase in link traffic volume will normally decrease the travel speed due to congestion and hence increase the travel time

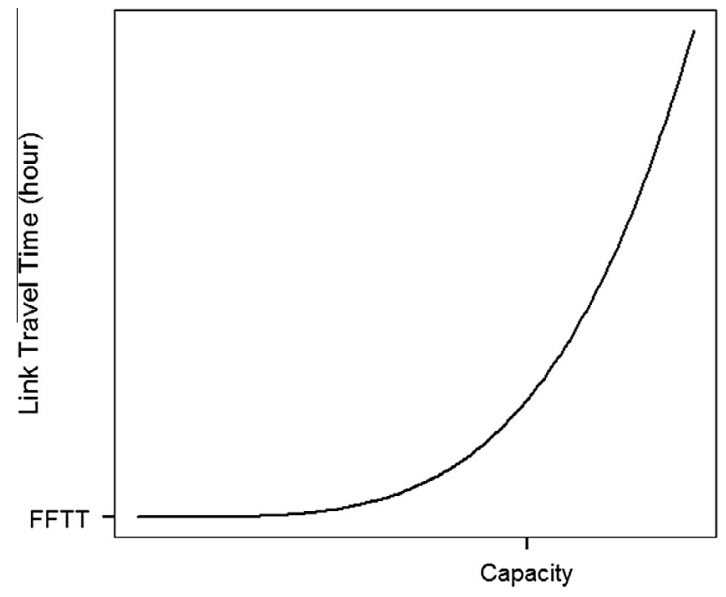

Link Flow Rate (veh/hour)

Fig. 1. A typical link performance function. 
along the link. Link travel time functions are also referred to as link performance functions, link capacity functions, volumedelay curves, link impedance functions and latency functions. In his study Branston (1976) investigates the link capacity functions in the literature reviewing more than 20 of them. A typical link performance function is shown in Fig. 1, where FFTT stands for the free flow travel time.

U.S. Department of Commerce Bureau of Public Roads expresses the relationship between travel time (or speed) and the volume of traffic on a link by the following function (refered to as the BPR function):

$$
t(x)=t^{0}\left(1+\alpha\left(\frac{x}{c}\right)^{\beta}\right)
$$

where $t(x)$ is the travel time at which assigned volume $x$ can travel on the link, $c$ is the practical capacity (maximum flow rate) and $t^{0}$ is the base travel time or free flow travel time at zero volume. The parameters $\alpha \geqslant 0$ and $\beta \geqslant 0$ are the tuning parameters defined in accordance with the road characteristics and they are taken as 0.15 and 4 by the U.S. Department of Commerce Bureau of Public Roads, respectively (TAM, 1964).

\subsection{Problem formulation under CSO traffic assignment model}

We define our problem on a directed network $G=(N, A)$, where $N$ is the set of nodes and $A$ is the set of arcs (links) in the network. Each $\operatorname{arc} a$ is associated with a convex travel time function (BPR function) $t_{a}$. We define $O$ as the set of origin (demand) nodes from where the evacuees at risk are to be evacuated and $F$ as the set of destination nodes (potential shelter sites) where evacuees reach to safety. Without loss of generality, we assume that $O$ and $F$ are disjoint subsets of $N$. The amount of demand at origin $r \in O, w_{r}$, is the number of passenger vehicles that will be evacuated. We denote the set of alternative paths between origin-destination pair $r-s$ by $P_{r s}$. The values $d_{r s}^{*}$ is the shortest path distance from demand node $r$ to shelter site $s$. The number $p$ is a predetermined parameter that restricts the number of shelter sites that can be opened due to budgetary and/or management issues.

We denote a driver's level of tolerance (or indifference) by $\lambda$. In other words, $\lambda$ is the level that a driver perceives two paths as equal to each other. We define the set of shortest paths from origin $r$ to destination $s$ of tolerance degree $\lambda$ as: $P_{r s}^{\lambda}=\left\{\pi \in P_{r s}: d^{\pi} \leqslant(1+\lambda) d_{r s}^{*}\right\}$, where $d^{\pi}$ is the length of path $\pi$ (one can also define this set based on free flow travel times if these reflect better the drivers' behavior). We compute this set using an algorithm developed by Byers and Waterman (1984).

The aim of our evacuation planning problem is to decide on the locations of $p$ shelters and the assignment of evacuees to shelters and routes so that the region is evacuated as quickly as possible.

We define the following variables to formulate the problem with CSO traffic assignment: $v_{\pi}$ is the fraction of demand that uses path $\pi \in P_{r s}^{\lambda}$ from origin $r \in O$ to destination $s \in F ; x_{a}$ is the number of vehicles on arc $a \in A$; the binary variable $y_{s}$ is 1 if a shelter site is opened at node $s \in F, 0$ otherwise. Using these variables, we formulate the evacuation planning problem as follows.

\section{Model CSO}

$$
\begin{array}{ll}
\min \sum_{a \in A} t_{a}^{0}\left(1+\alpha\left(\frac{x_{a}}{c_{a}}\right)^{\beta}\right) x_{a} & \\
\text { s.t. } \sum_{s \in F} \sum_{\pi \in P_{r s}^{\prime}} v_{\pi}^{\prime}=1 & \forall r \in O, \\
\sum_{\pi \in P_{r s}^{i}} v_{\pi} \leqslant y_{s} & \forall r \in O, s \in F, \\
\sum_{s \in F} y_{s}=p, & \\
\sum_{s \in F} \sum_{\pi \in P_{r s}^{\lambda}: d^{\pi}>(1+\lambda) d_{r i}^{*}} v_{\pi}+y_{i} \leqslant 1 & \forall r \in O, i \in F, \\
x_{a}=\sum_{r \in O} \sum_{s \in F} \sum_{\pi \in P_{r s}^{\prime}: a \in \pi} w_{r} v_{\pi} & \forall a \in A, \\
v_{\pi} \geqslant 0 & \forall \pi \in \cup_{r \in O, s \in F} P \\
y_{s} \in\{0,1\} & \forall s \in F .
\end{array}
$$

Objective function (1) minimizes the total travel time that evacuees spend in the network. Constraints (2) ensure that all demand is evacuated. Constraints (3) forbid assigning demand to non-open shelter sites. Constraint (4) limits the number of shelter sites open to a pre-specified number $p$. Constraints (5) ensure that if the shelter at site $i$ is open, then the demand at origin node $r$ cannot be routed on any path whose length is longer than $(1+\lambda) d_{r i}^{*}$. The set of constraints (6) computes the traffic flow on every arc and constraints (7) and (8) are variable restrictions.

If the central authority planning the evacuation requires the entire demand at a given origin to be routed on the same path to the same shelter, then one can define the variables $v_{\pi}$ as binary variables. 
Evacuation management authority may also require the evacuees of the same origin to be allocated to the same shelter while allowing the traffic from an origin to a shelter site to be distributed between alternative routes. To enable having separate control levels over the assignment of demand to shelters and to alternative paths we define an additional variable $z_{r s}$, which takes value one if origin $r$ is assigned to shelter $s$ and zero otherwise. We add the constraints $\sum_{\pi \in P_{r s}^{\lambda}} v_{\pi}=z_{r s}$ for all $r \in O$ and $s \in F$.

Note that if $\alpha=0, G$ is a complete bipartite graph where $N=O \cup F$ and arcs go from nodes in $O$ to nodes in $F$, then our problem reduces to the $p$-median problem, which is NP-hard (Kariv and Hakimi, 1979). Hence, we can conclude that the evacuation planning problem under CSO traffic assignment model is NP-hard even when $\alpha=0$ and $G$ is bipartite.

The CSO approach generalizes both the SO and the NA traffic assignment approaches. When $\lambda=0$, the above formulation models the evacuation planning problem under the nearest allocation traffic assignment model. When $\lambda=\infty$, we obtain a model for the SO traffic assignment.

Finally, note that our model is different from a traffic assignment model for a given set of origin-destination flows since in our case, the origins are known and the destinations are decided optimally.

\subsection{Formulation for the SO traffic assignment model}

To compare the results of the CSO model, we also solve the same evacuation planning problems with SO traffic assignment model. The SO model decides on the locations of shelter sites and assigns the evacuees to shelters and routes so that the total evacuation time is minimized.

One can formulate the SO evacuation planning problem as done in Sherali et al. (1991). We use $\delta^{-}(i)$ and $\delta^{+}(i)$ to denote the sets of incoming and outgoing arcs of node $i \in N$, respectively. In addition to the variables defined above, we define $f_{s}$ to be the number of evacuees who arrive in shelter $s \in F$.

\section{Model So}

$$
\min \sum_{a \in A} t_{a}^{0}\left(1+\alpha\left(\frac{x_{a}}{c_{a}}\right)^{\beta}\right) x_{a}
$$

s.t. (4) and (8),

$$
\begin{array}{ll}
\sum_{a \in \delta^{-}(i)} x_{a}-\sum_{a \in \delta^{+}(i)} x_{a}= \begin{cases}-w_{i} & \forall i \in O \\
0 & \forall i \in N \backslash(O \cup F) \\
f_{i}, & \forall i \in F\end{cases} \\
0 \leqslant f_{s} \leqslant \sum_{r \in O} w_{r} y_{s} & \forall s \in F \\
x_{a} \geqslant 0 & \forall a \in A .
\end{array}
$$

Objective function (9) minimizes the total evacuation time. Constraints (10) are flow balance constraints. Finally, constraints (11) ensure that if a shelter site is not open, then no evacuee can be assigned to it.

We also use a multi-commodity version of this model to have the routes of evacuees in an optimal solution.

\subsection{Second order cone programming approach}

Most of the approaches for evacuation planning problems with a congestion related nonlinear objective are heuristics. Alternatively the nonlinear objective function may be approximated with a piecewise linear function. Here we avoid these two approaches. Motivated by the advances in second order cone programming (see, e.g., Nemirovski and Tal (2001) and Alizadeh and Goldfarb (2003)), we reformulate the nonlinear mixed integer programming models presented in the previous sections as second order conic mixed integer programs.

Gürel (2011) shows that a multi-commodity network flow problem with congestion and capacity expansion can be efficiently formulated by using second order conic programming. He states that his approach can be adopted to problems with the BPR function.

We first define auxiliary variables $\mu_{a}$ for each $a \in A$ and move the nonlinearity from the objective function to the constraints, i.e., the objective function of the CSO model becomes $\sum_{a \in A}\left(t_{a}^{0} x_{a}+\frac{t_{a}^{0} \alpha}{c_{a}^{\beta}} \mu_{a}\right)$ and we add the constraints $x_{a}^{\beta+1} \leqslant \mu_{a}$ for all $a \in A$.

We take $\beta=4$ and represent $x_{a}^{5} \leqslant \mu_{a}$ with

$$
\begin{aligned}
& x_{a}^{2} \leqslant \theta_{a} h_{a}, \\
& \theta_{a}^{2} \leqslant u_{a} x_{a}, \\
& u_{a}^{2} \leqslant \mu_{a} x_{a}, \\
& h_{a}=1, \theta_{a}, u_{a}, x_{a}, \mu_{a} \geqslant 0 .
\end{aligned}
$$


And these hyperbolic inequalities are represented by their respective quadratic cone constraints:

$$
\begin{aligned}
& \left\|2 x_{a}, \theta_{a}-h_{a}\right\| \leqslant \theta_{a}+h_{a}, \\
& \left\|2 \theta_{a}, u_{a}-x_{a}\right\| \leqslant u_{a}+x_{a}, \\
& \left\|2 u_{a}, \mu_{a}-x_{a}\right\| \leqslant \mu_{a}+x_{a}, \\
& h_{a}=1, \theta_{a}, u_{a}, x_{a}, \mu_{a} \geqslant 0 .
\end{aligned}
$$

\section{Computational study}

\subsection{Instances}

We solved the models CSO and SO with different test networks. The test problems we used are from three sources. The first source is an online library called Transportation Network Test Problems (TNTP, 2001) and the second is the OR-Library (OR-Library, 1990), originally described in (Beasley, 1990). We got the data for Istanbul road network from the masters thesis of Kırıkçı (2012) who worked in collaboration with the Disaster Coordination Center of Istanbul Metropolitan Municipality. Computational tests were performed on a laptop with a $2.4 \mathrm{GHz}$. Intel i7-3630QM CPU and $16 \mathrm{~GB}$ of RAM using Java ILOG CPLEX version 12.5.1.

As geographical distances and free flow travel times are highly correlated (Jahn et al., 2005), we used the geographical distances as arc lengths in our analysis. We employed the standard BPR function and assumed that the parameters of the function are the same for all arcs (road segments), i.e., $\alpha=0.15$ and $\beta=4$ for all $a \in A$.

Sioux Falls and Anaheim networks were downloaded from (TNTP, 2001). In these instances, the demands are for origindestination pairs. We take the demand at node $r$ as the sum of the demands whose origin is node $r$. For Sioux Falls we also performed runs with demand at each origin $r \in O$ as one tenth of the original demand. The original and modified instances are referred to as Sioux Falls 1 and 2, respectively.

We downloaded the P-median1 and P-median6 instances from the (OR-Library, 1990) and used their network structure. We created the demand for each origin node randomly between 1000 and 2000 . We also generated potential shelters sites randomly on the network for these instances. We assumed all arcs (road segments) have two lanes and three lanes for Pmedian1 and P-median6 instances, respectively, with a maximum traffic flow rate (capacity) of 2000 vehicles per hour per lane and with a free flow speed of $80 \mathrm{~km} / \mathrm{h}$ in an uninterrupted traffic flow.

Istanbul houses a population of more than 14 million people, which is above one-sixth of the total population of Turkey (TÜiK, 2013). In addition to the high earthquake hazard of the city, the risk for earthquake has increased due to the improper land-use planning, construction, overpopulation and other reasons (Erdik and Durukal, 2008). The efforts for earthquake preparedness and response planning for an impending major earthquake in Istanbul were motivated by the massively destructive 1999 Marmara (Turkey) earthquake, followed by a disaster prevention and mitigation study conducted by the Istanbul Metropolitan Municipality (IMM) in collaboration with the Japan International Cooperation Agency (JICA) (IMMJICA, 2002). The report by IMM and JICA points out that it is imperative that a community evacuation system be established. For the Istanbul network, we assumed that each road segment has three lanes with a maximum flow rate of 2000 vehicles per hour per lane and with a free flow speed of $90 \mathrm{~km} / \mathrm{h}$. Each vehicle was assumed to carry three passengers on the average. In the report by the IMM and JICA (IMM-JICA, 2002), it is stated that all residents in heavily damaged buildings, half of the residents in moderately damaged buildings and $10 \%$ of the residents in partially damaged buildings adds up to 1.3 million citizens who require shelters in accordance with the most probable scenario. A similar number is given for each district of Istanbul by Kırıkçı (2012). We assumed that only those people in need of a shelter will be evacuated. The potential shelter sites are determined by interviews with the IMM as stated by Kırıç̧ı (2012). As there are two bridges that connect the European and Anatolian sides of Istanbul City, we assumed that the population living on each side of the Bosphorus will be evacuated to shelters on their own side.

The specifics of the instances used in the computational study are shown in Table 1 . Here $|O-F|$ is the number of origin

\begin{tabular}{|c|c|c|c|c|c|c|}
\hline Instance & $|N|$ & $|A|$ & $|O|$ & $|F|$ & Total demand & $|O-F|$ \\
\hline Sioux Falls 1 & 24 & 76 & 15 & 9 & 234,600 & 135 \\
\hline Sioux Falls 2 & 24 & 76 & 15 & 9 & 23,460 & 135 \\
\hline P-median1 & 100 & 396 & 90 & 10 & 132,212 & 900 \\
\hline P-median6 & 200 & 1572 & 176 & 24 & 260,520 & 4224 \\
\hline Anaheim & 416 & 914 & 37 & 17 & 104,698 & 408 \\
\hline Istanbul Anatolian & 124 & 362 & 13 & 17 & 110,843 & 221 \\
\hline Istanbul European & 158 & 448 & 25 & 32 & 363,865 & 800 \\
\hline
\end{tabular}
destination pairs that are connected with a directed path.

Table 1

Specifics of the instances used in the computational study. 


\subsection{Computational performance}

In Tables 2 and 3, we report the results of the CSO model. For each instance, we report the number of paths in the network, the number of paths with positive flow in the optimal solution, the gap between the optimal value and the continuous relaxation at the root node, the number of nodes enumerated and the solution time in seconds. All instances are solved to optimality and the longest computation time is slightly more than half an hour. We observe that, in general, the gap, the number of nodes enumerated and the solution time decrease with increasing $p$. We also observe that even though the gaps tend to decrease as $\lambda$ increases, the solution times increase as the number of paths increase. If $p$ is not very small or very large and if $\lambda>0$, then the number of paths with positive flow decreases as $p$ increases and the gap and the solution times also decrease. For Sioux Falls 1 with $\lambda=0.2$, the computation time increases when we increase $p$ from four to five. The same

Table 2

Computational performance I.

\begin{tabular}{|c|c|c|c|c|c|c|c|}
\hline$p$ & $\lambda$ & \# of paths & $\begin{array}{l}\text { \# of paths with } \\
\text { positive flow }\end{array}$ & Gap & Nodes & Total evacuation time & Solution time \\
\hline \multicolumn{8}{|c|}{ Sioux Falls 1} \\
\hline 2 & 0 & 138 & 15 & 66.74 & 32 & $18,050,148$ & 1.23 \\
\hline 3 & 0 & 138 & 17 & 49.04 & 46 & $9,363,128$ & 1.46 \\
\hline 4 & 0 & 138 & 16 & 50.96 & 23 & $9,497,033$ & 1.82 \\
\hline 5 & 0 & 138 & 15 & 34.69 & 8 & $7,556,851$ & 1.56 \\
\hline 7 & 0 & 138 & 16 & 0.00 & 0 & $8,122,617$ & 0.14 \\
\hline 9 & 0 & 138 & 16 & 0.00 & 0 & $76,375,938$ & 0.07 \\
\hline 2 & 0.1 & 214 & 20 & 80.52 & 43 & $15,040,993$ & 1.81 \\
\hline 3 & 0.1 & 214 & 20 & 60.47 & 30 & $8,550,802$ & 2.05 \\
\hline 4 & 0.1 & 214 & 16 & 52.24 & 37 & $9,497,033$ & 1.73 \\
\hline 5 & 0.1 & 214 & 15 & 37.22 & 20 & $7,556,851$ & 1.54 \\
\hline 7 & 0.1 & 214 & 16 & 22.59 & 3 & $8,122,617$ & 1.36 \\
\hline 9 & 0.1 & 214 & 16 & 0.00 & 0 & $76,375,938$ & 0.09 \\
\hline 2 & 0.2 & 389 & 33 & 81.37 & 44 & $4,852,731$ & 2.47 \\
\hline 3 & 0.2 & 389 & 23 & 71.71 & 29 & $3,242,163$ & 1.65 \\
\hline 4 & 0.2 & 389 & 25 & 52.89 & 27 & $2,109,087$ & 1.47 \\
\hline 5 & 0.2 & 389 & 26 & 45.93 & 28 & $1,998,505$ & 2.09 \\
\hline 7 & 0.2 & 389 & 22 & 4.38 & 2 & $4,081,764$ & 1.52 \\
\hline 9 & 0.2 & 389 & 21 & 0.00 & 0 & $74,137,933$ & 0.07 \\
\hline \multicolumn{8}{|c|}{$P$-median1 } \\
\hline 2 & 0 & 906 & 90 & 97.52 & 38 & $1,276,280$ & 12.21 \\
\hline 5 & 0 & 906 & 90 & 91.75 & 59 & 306,667 & 7.87 \\
\hline 8 & 0 & 906 & 90 & 82.11 & 15 & 255,896 & 5.23 \\
\hline 10 & 0 & 906 & 90 & 0.00 & 0 & 258,510 & 0.16 \\
\hline 2 & 0.1 & 1450 & 94 & 96.54 & 45 & 692,957 & 14.11 \\
\hline 5 & 0.1 & 1450 & 102 & 67.80 & 56 & 57,023 & 8.83 \\
\hline 8 & 0.1 & 1450 & 105 & 37.99 & 10 & 35,674 & 4.82 \\
\hline 10 & 0.1 & 1450 & 97 & 0.00 & 0 & 39,708 & 0.18 \\
\hline 2 & 0.2 & 2450 & 92 & 91.63 & 49 & 269,051 & 17.05 \\
\hline 5 & 0.2 & 2450 & 136 & 48.30 & 116 & 34,165 & 10.90 \\
\hline 8 & 0.2 & 2450 & 137 & 20.50 & 31 & 23,371 & 7.08 \\
\hline 10 & 0.2 & 2450 & 125 & 0.00 & 0 & 24,881 & 0.27 \\
\hline 2 & 0.5 & 13,006 & 118 & 77.12 & 83 & 90,884 & 38.56 \\
\hline 5 & 0.5 & 13,006 & 235 & 21.19 & 120 & 21,793 & 23.05 \\
\hline 8 & 0.5 & 13,006 & 220 & 5.50 & 32 & 16,842 & 8.96 \\
\hline 10 & 0.5 & 13,006 & 193 & 0.00 & 0 & 15,864 & 0.46 \\
\hline \multicolumn{8}{|c|}{ P-median6 } \\
\hline 5 & 0 & 4335 & 176 & 71.55 & 893 & 54,386 & 325.92 \\
\hline 10 & 0 & 4335 & 178 & 27.09 & 1016 & 18,002 & 111.47 \\
\hline 15 & 0 & 4335 & 176 & 17.11 & 448 & 14,753 & 35.18 \\
\hline 20 & 0 & 4335 & 177 & 16.09 & 107 & 14,288 & 18.07 \\
\hline 5 & 0.1 & 8160 & 194 & 45.33 & 1563 & 28,225 & 445.71 \\
\hline 10 & 0.1 & 8160 & 194 & 13.75 & 844 & 15,122 & 97.43 \\
\hline 15 & 0.1 & 8160 & 191 & 8.30 & 156 & 13,120 & 22.50 \\
\hline 20 & 0.1 & 8160 & 197 & 8.19 & 54 & 12,660 & 11.70 \\
\hline 5 & 0.2 & 15,896 & 221 & 29.62 & 1499 & 21,827 & 607.14 \\
\hline 10 & 0.2 & 15,896 & 228 & 9.60 & 1351 & 14,484 & 154.00 \\
\hline 15 & 0.2 & 15,896 & 221 & 5.69 & 728 & 12,728 & 41.82 \\
\hline 20 & 0.2 & 15,896 & 223 & 3.88 & 18 & 11,976 & 13.18 \\
\hline 5 & 0.4 & 63,471 & 264 & 18.61 & 2052 & 18,934 & $1,066.59$ \\
\hline 10 & 0.4 & 63,471 & 278 & 8.14 & 1937 & 14,203 & 412.40 \\
\hline 15 & 0.4 & 63,471 & 260 & 3.07 & 654 & 12,293 & 85.96 \\
\hline 20 & 0.4 & 63,471 & 219 & 0.86 & 27 & 11,465 & 17.00 \\
\hline
\end{tabular}


Table 3

Computational performance II.

\begin{tabular}{|c|c|c|c|c|c|c|c|}
\hline$p$ & $\lambda$ & \# of paths & $\begin{array}{l}\text { \# of paths with } \\
\text { positive flow }\end{array}$ & Gap & Nodes & Total evacuation time & Solution time \\
\hline \multicolumn{8}{|c|}{ Anaheim } \\
\hline 5 & 0 & 602 & 26 & 56.92 & 802 & 29,140 & 8.58 \\
\hline 8 & 0 & 602 & 26 & 54.26 & 373 & 28,334 & 22.62 \\
\hline 10 & 0 & 602 & 26 & 51.20 & 333 & 28,334 & 14.53 \\
\hline 12 & 0 & 602 & 26 & 44.44 & 98 & 28,335 & 7.11 \\
\hline 15 & 0 & 602 & 26 & 34.00 & 14 & 29,852 & 3.38 \\
\hline 5 & 0.1 & 44,789 & 44 & 37.51 & 347 & 15,511 & 49.05 \\
\hline 8 & 0.1 & 44,789 & 46 & 35.08 & 238 & 14,895 & 22.86 \\
\hline 10 & 0.1 & 44,789 & 44 & 31.84 & 179 & 14,815 & 28.09 \\
\hline 12 & 0.1 & 44,789 & 33 & 23.37 & 44 & 15,239 & 16.22 \\
\hline 15 & 0.1 & 44,789 & 32 & 48.02 & 16 & 24,738 & 10.74 \\
\hline 5 & 0.2 & 787,198 & 78 & 24.45 & 276 & 11,892 & $1,907.59$ \\
\hline 8 & 0.2 & 787,198 & 71 & 22.14 & 272 & 11,615 & $1,618.45$ \\
\hline 10 & 0.2 & 787,198 & 79 & 20.42 & 179 & 11,594 & 457.89 \\
\hline 12 & 0.2 & 787,198 & 73 & 20.50 & 138 & 11,630 & 171.78 \\
\hline 15 & 0.2 & 787,198 & 40 & 5.76 & 13 & 12,324 & 66.24 \\
\hline \multicolumn{8}{|c|}{ Istanbul European } \\
\hline 12 & 0 & 815 & 25 & 88.09 & 1300 & $1,080,069$ & 13.68 \\
\hline 17 & 0 & 815 & 25 & 87.86 & 1064 & $1,063,162$ & 10.79 \\
\hline 22 & 0 & 815 & 25 & 87.99 & 1290 & $1,062,640$ & 25.21 \\
\hline 27 & 0 & 815 & 25 & 86.77 & 358 & $1,062,560$ & 8.91 \\
\hline 32 & 0 & 815 & 25 & 0.01 & 0 & $1,070,171$ & 0.16 \\
\hline 12 & 0.1 & 33,723 & 36 & 91.05 & 3,487 & $1,065,615$ & 125.12 \\
\hline 17 & 0.1 & 33,723 & 30 & 90.99 & 2,026 & $1,055,381$ & 63.81 \\
\hline 22 & 0.1 & 33,723 & 29 & 90.99 & 1763 & $1,054,913$ & 55.07 \\
\hline 27 & 0.1 & 33,723 & 29 & 89.54 & 590 & $1,054,888$ & 18.39 \\
\hline 32 & 0.1 & 33,723 & 26 & 0.00 & 0 & $1,070,163$ & 0.56 \\
\hline 12 & 0.15 & 225,449 & 52 & 78.62 & 1705 & 445,586 & 392.41 \\
\hline 17 & 0.15 & 225,449 & 41 & 78.19 & 728 & 433,460 & 157.14 \\
\hline 22 & 0.15 & 225,449 & 38 & 78.08 & 627 & 432,234 & 137.79 \\
\hline 27 & 0.15 & 225,449 & 38 & 76.73 & 381 & 432,239 & 129.89 \\
\hline 32 & 0.15 & 225,449 & 29 & 0.01 & 0 & 462,698 & 3.29 \\
\hline \multicolumn{8}{|c|}{ Istanbul Anatolian } \\
\hline 7 & 0 & 221 & 13 & 67.44 & 109 & 58,731 & 5.00 \\
\hline 10 & 0 & 221 & 13 & 68.20 & 60 & 57,446 & 3.24 \\
\hline 13 & 0 & 221 & 13 & 64.78 & 51 & 57,445 & 3.52 \\
\hline 15 & 0 & 221 & 13 & 44.77 & 18 & 57,445 & 2.88 \\
\hline 7 & 0.1 & 7151 & 20 & 75.10 & 124 & 55,404 & 7.29 \\
\hline 10 & 0.1 & 7151 & 20 & 74.08 & 62 & 54,254 & 5.66 \\
\hline 13 & 0.1 & 7151 & 18 & 67.91 & 39 & 54,173 & 4.48 \\
\hline 15 & 0.1 & 7151 & 18 & 42.80 & 13 & 54,173 & 4.55 \\
\hline 7 & 0.2 & 133,183 & 29 & 52.51 & 62 & 26,460 & 18.03 \\
\hline 10 & 0.2 & 133,183 & 26 & 54.50 & 41 & 25,992 & 13.61 \\
\hline 13 & 0.2 & 133,183 & 28 & 49.89 & 21 & 25,992 & 12.70 \\
\hline 15 & 0.2 & 133,183 & 21 & 32.60 & 11 & 51,054 & 12.17 \\
\hline 7 & 0.3 & $1,123,027$ & 39 & 48.36 & 138 & 23,423 & 207.10 \\
\hline 10 & 0.3 & $1,123,027$ & 39 & 46.54 & 78 & 22,956 & 724.21 \\
\hline 13 & 0.3 & $1,123,027$ & 41 & 46.06 & 52 & 22,957 & $1,427.39$ \\
\hline 15 & 0.3 & $1,123,027$ & 24 & 56.62 & 13 & 49,475 & 263.41 \\
\hline
\end{tabular}

happens for Istanbul Anatolian network with $\lambda=0.3$ when $p$ is increased from 10 to 13 and in both cases the number of paths with positive flow also increases.

\subsection{The impact of the number and locations of shelters on the total evacuation time}

In Tables 2 and 3, we also report the total evacuation time for all instances. It is interesting to see that increasing the number of shelters improves the system performance up to a point. For example, for Sioux Falls 1 instance, when $p$ is four and the tolerance level is 0.2 , the total evacuation time is about two million hours and when we increase $p$ to seven and nine, the total evacuation time increases to more than four million and 74 million hours, respectively. Fig. 2 illustrates how this happens. The potential shelter sites are at nodes $2,6-8,16-20$. When $p$ is four, the demands at nodes 9,10 and 11 are assigned to two different shelter sites ( 8 and 19) through eight different routes. But when $p$ is nine, the new shelter site 16 is much closer to those demand nodes than others, so nodes 9, 10, 11 all get assigned to shelter site 16 and the evacuees at each of these nodes use a single path to reach shelter 16 . The total demand at these three nodes constitutes approximately $35.7 \%$ of the total evacuation demand and the three paths used to reach the shelter 16 share a common arc, $(10,16)$, which causes a bottleneck due to over 

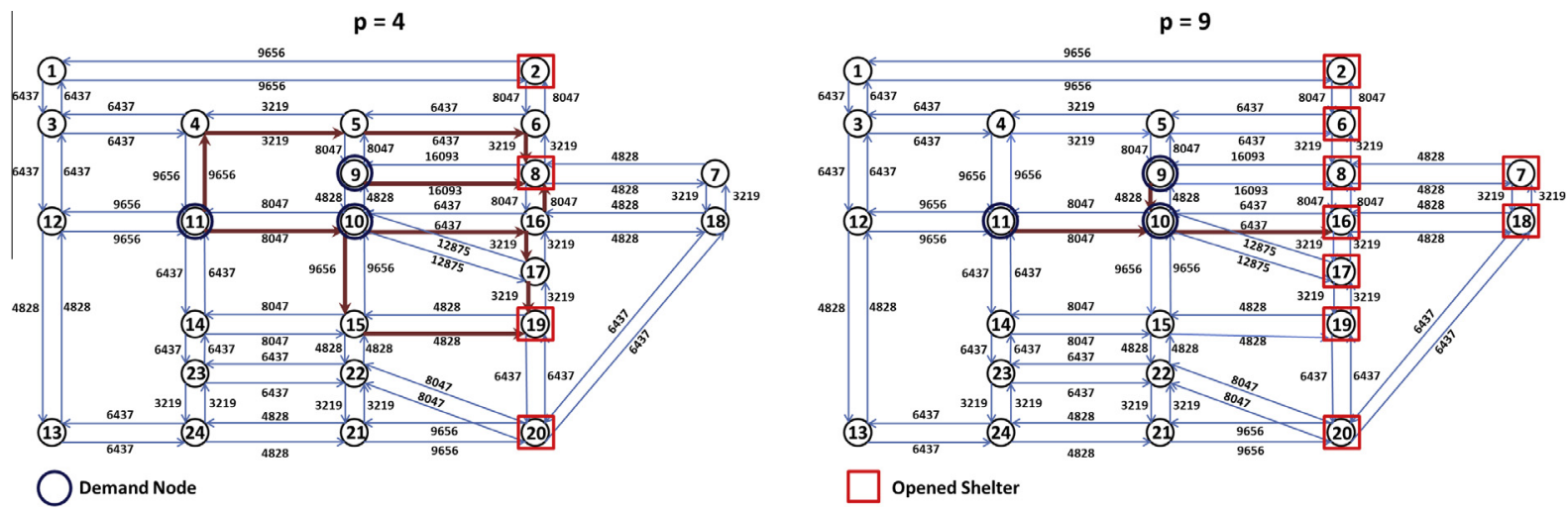

Fig. 2. Sioux Falls 1: Allocation of demand nodes when $p=4$ and $p=9$.

congestion, thus increasing the total evacuation time. This example shows that the choice of potential shelter locations and the number of shelters to open is critical for the efficiency of an evacuation plan. Clearly, using distances instead of real travel times in modeling evacuees' choices may result in a congested system as seen in the above example. However, as pointed out in the Introduction, it is not reasonable to assume that the evacuees have full information on travel times on every possible route. Instead, one may try to estimate the congested travel times and use them as normal lengths.

Fig. 3 depicts the effect of the number of shelters and the level of tolerance on the total evacuation time for Sioux Falls 1 and 2. We observe that when the network is overloaded, which is the case of Sioux Falls 1, increasing the number of shelters to nine has an adverse effect for all tolerance levels. We also observe a similar behavior for Sioux Falls 2 when $\lambda$ is small. For this instance, when $\lambda \geqslant 0.3$, we do not observe an adverse effect when the number of shelters is increased to nine, however there is no improvement. We also observe that when $p$ is two, the change in the level of tolerance has little or no effect on the total evacuation time for Sioux Falls 2 whereas an opposite result is observed for Sioux Falls 1.

\subsection{Efficiency and fairness}

While deciding on the number and location of shelters and assigning evacuees to shelters and to routes, our goal is to establish an efficient evacuation plan without losing fairness among evacuees. We measure the efficiency of an evacuation plan with regard to performance criteria such as the total evacuation time and the maximum latency.

The price of anarchy measures the impact of selfishness. In the literature (Koutsoupias and Papadimitriou, 1999; Roughgarden, 2002; Jahn et al., 2003; Schulz and Moses, 2003; Correa et al., 2005; Schulz and Stier-Moses, 2006; Correa et al., 2007; Olsthoorn, 2012), it is defined as the worst possible proportion between the social utility from a user equilibrium and the system optimal. In our setting, we do not have evacuees acting on their own, but the system compromises efficiency for fairness. Hence we define price of fairness to measure the difference between the total evacuation times of our CSO solutions and the SO solution. Let $\tau_{\mathrm{CSO}}(\lambda)$ and $\tau_{\mathrm{SO}}$ be the optimal total evacuation times for the CSO model with $\lambda$ level of tolerance and the SO model, respectively. The price of fairness for tolerance level $\lambda$ is
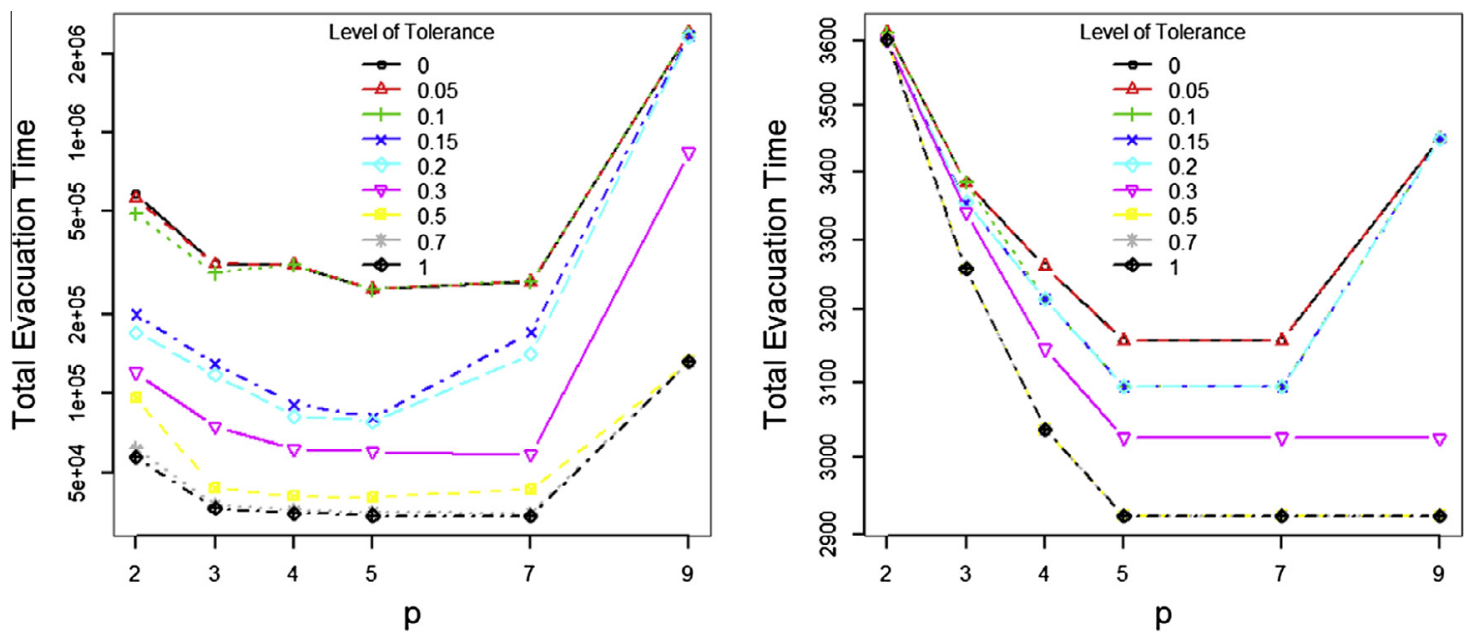

Fig. 3. The effect of $p$ and the level of tolerance on the total evacuation time, Sioux Falls 1 and 2. 
Table 4

Efficiency and fairness I.

\begin{tabular}{|c|c|c|c|c|c|c|c|c|}
\hline$p$ & $\lambda$ & Total evacuation time & $\rho(\lambda)$ & ML & NUR & LUR & NUS & LUS \\
\hline \multicolumn{9}{|c|}{ Sioux Falls 1} \\
\hline \multirow[t]{6}{*}{3} & SO & 484,808 & - & 2.457 & 3.000 & 1.092 & 4.125 & 1.161 \\
\hline & 0 & $9,363,128$ & 19.313 & 78.764 & 1.000 & 1.000 & 1.000 & 1.003 \\
\hline & 0.05 & $9,363,063$ & 19.313 & 78.764 & 1.000 & 1.000 & 1.000 & 1.003 \\
\hline & 0.1 & $8,550,802$ & 17.638 & 78.376 & 1.083 & 1.003 & 1.083 & 1.013 \\
\hline & 0.15 & $3,634,100$ & 7.496 & 20.673 & 1.125 & 1.001 & 1.125 & 1.001 \\
\hline & 0.2 & $3,242,163$ & 6.688 & 17.776 & 1.182 & 1.002 & 1.200 & 1.002 \\
\hline \multirow[t]{6}{*}{5} & SO & 472,219 & - & 2.423 & 5.250 & 1.116 & 6.000 & 1.163 \\
\hline & 0 & $7,556,851$ & 16.003 & 75.106 & 1.000 & 1.000 & 1.000 & 1.000 \\
\hline & 0.05 & $7,556,851$ & 16.003 & 75.106 & 1.000 & 1.000 & 1.000 & 1.000 \\
\hline & 0.1 & $7,556,851$ & 16.003 & 75.106 & 1.000 & 1.000 & 1.000 & 1.000 \\
\hline & 0.15 & $2,107,745$ & 4.463 & 18.447 & 1.125 & 1.001 & 1.125 & 1.002 \\
\hline & 0.2 & $1,998,505$ & 4.232 & 12.049 & 1.167 & 1.002 & 1.200 & 1.003 \\
\hline \multicolumn{9}{|c|}{ Sioux Falls 2} \\
\hline \multirow[t]{6}{*}{3} & SO & 3258 & - & 0.282 & 1.250 & 1.190 & 1.333 & 1.242 \\
\hline & 0 & 3383 & 1.038 & 0.243 & 1.000 & 1.000 & 1.000 & 1.000 \\
\hline & 0.05 & 3383 & 1.038 & 0.243 & 1.000 & 1.000 & 1.000 & 1.000 \\
\hline & 0.1 & 3383 & 1.038 & 0.243 & 1.000 & 1.000 & 1.000 & 1.000 \\
\hline & 0.15 & 3354 & 1.030 & 0.269 & 1.125 & 1.097 & 1.143 & 1.110 \\
\hline & 0.2 & 3354 & 1.030 & 0.269 & 1.125 & 1.097 & 1.200 & 1.153 \\
\hline \multirow[t]{6}{*}{5} & SO & 2923 & - & 0.232 & 1.000 & 1.000 & 1.330 & 1.247 \\
\hline & 0 & 3157 & 1.080 & 0.232 & 1.000 & 1.000 & 1.000 & 1.000 \\
\hline & 0.05 & 3157 & 1.080 & 0.232 & 1.000 & 1.000 & 1.000 & 1.000 \\
\hline & 0.1 & 3094 & 1.058 & 0.232 & 1.000 & 1.000 & 1.091 & 1.000 \\
\hline & 0.15 & 3094 & 1.058 & 0.232 & 1.000 & 1.000 & 1.111 & 1.087 \\
\hline & 0.2 & 3094 & 1.058 & 0.232 & 1.182 & 1.000 & 1.200 & 1.154 \\
\hline \multicolumn{9}{|c|}{ P-median1 } \\
\hline \multirow[t]{6}{*}{5} & SO & 20,821 & - & 0.417 & 5.500 & 2.503 & 11.125 & 2.816 \\
\hline & 0 & 306,667 & 14.729 & 8.271 & 1.000 & 1.000 & 1.000 & 1.000 \\
\hline & 0.05 & 88,666 & 4.259 & 2.387 & 1.046 & 1.000 & 1.046 & 1.044 \\
\hline & 0.1 & 57,023 & 2.739 & 0.864 & 1.085 & 1.016 & 1.099 & 1.021 \\
\hline & 0.15 & 37,472 & 1.800 & 0.525 & 1.135 & 1.039 & 1.148 & 1.046 \\
\hline & 0.2 & 34,165 & 1.641 & 0.487 & 1.184 & 1.055 & 1.200 & 1.085 \\
\hline \multirow[t]{6}{*}{8} & SO & 16,202 & - & 0.266 & 1.611 & 1.399 & 11.125 & 3.377 \\
\hline & 0 & 255,896 & 15.794 & 7.822 & 1.000 & 1.000 & 1.000 & 1.000 \\
\hline & 0.05 & 48,139 & 2.971 & 1.037 & 1.000 & 1.000 & 1.050 & 1.002 \\
\hline & 0.1 & 35,674 & 2.202 & 0.617 & 1.085 & 1.007 & 1.099 & 1.027 \\
\hline & 0.15 & 24,081 & 1.486 & 0.344 & 1.135 & 1.071 & 1.148 & 1.079 \\
\hline & 0.2 & 23,371 & 1.442 & 0.344 & 1.151 & 1.072 & 1.200 & 1.096 \\
\hline \multicolumn{9}{|c|}{ P-median6 } \\
\hline \multirow[t]{6}{*}{10} & SO & 14,121 & - & 0.132 & 1.514 & 1.359 & 4.000 & 2.221 \\
\hline & 0 & 18,002 & 1.275 & 0.173 & 1.000 & 1.000 & 1.000 & 1.000 \\
\hline & 0.05 & 16,078 & 1.139 & 0.134 & 1.033 & 1.025 & 1.043 & 1.029 \\
\hline & 0.1 & 15,122 & 1.071 & 0.140 & 1.091 & 1.057 & 1.100 & 1.057 \\
\hline & 0.15 & 14,684 & 1.040 & 0.127 & 1.139 & 1.097 & 1.146 & 1.111 \\
\hline & 0.2 & 14,484 & 1.026 & 0.129 & 1.161 & 1.124 & 1.200 & 1.147 \\
\hline \multirow[t]{6}{*}{15} & SO & 12,239 & - & 0.127 & 1.282 & 1.000 & 2.600 & 1.964 \\
\hline & 0 & 14,753 & 1.205 & 0.154 & 1.000 & 1.000 & 1.000 & 1.000 \\
\hline & 0.05 & 13,524 & 1.105 & 0.131 & 1.015 & 1.011 & 1.048 & 1.037 \\
\hline & 0.1 & 13,120 & 1.072 & 0.126 & 1.041 & 1.011 & 1.100 & 1.060 \\
\hline & 0.15 & 12,928 & 1.056 & 0.127 & 1.139 & 1.101 & 1.145 & 1.109 \\
\hline & 0.2 & 12,728 & 1.040 & 0.130 & 1.161 & 1.126 & 1.200 & 1.145 \\
\hline
\end{tabular}

$$
\rho(\lambda)=\frac{\tau_{\text {CSO }}(\lambda)}{\tau_{S O}} .
$$

We need to be fair to evacuees in two ways; first with respect to the travel times to their shelter sites and second with respect to the lengths of the routes they take. We employ two unfairness notions defined by Jahn et al. (2005), namely, normal unfairness and loaded unfairness. Let $F^{*}$ be the set of open shelter sites and $v^{*}$ be the routing in an optimal solution.

Normal unfairness with respect to routes: Ratio of the length of an evacuee's route to the length of the shortest route for the same origin-destination pair, both measured with respect to normal arc lengths:

$$
N U R=\max _{r \in O, s \in F^{*}} \max _{\pi \in P_{r s}^{i}: v_{\pi}^{*}>0} \frac{d^{\pi}}{d_{r s}^{*}} .
$$

Normal unfairness with respect to shelters: Ratio of the length of an evacuee's route to a shelter site, to the length of the shortest route to the nearest shelter site for the same origin, both measured with respect to normal arc lengths: 
Table 5

Efficiency and fairness II.

\begin{tabular}{|c|c|c|c|c|c|c|c|c|}
\hline$p$ & $\lambda$ & Total evacuation time & $\rho(\lambda)$ & ML & NUR & LUR & NUS & LUS \\
\hline \multicolumn{9}{|c|}{ Anaheim } \\
\hline \multirow[t]{6}{*}{10} & so & 8490 & - & 0.180 & 2.286 & 1.794 & 2.633 & 1.813 \\
\hline & 0 & 28,334 & 3.337 & 1.203 & 1.000 & 1.000 & 1.000 & 1.103 \\
\hline & 0.05 & 20,104 & 2.368 & 0.556 & 1.000 & 1.000 & 1.047 & 1.198 \\
\hline & 0.1 & 14,815 & 1.745 & 0.415 & 1.096 & 1.146 & 1.099 & 1.149 \\
\hline & 0.15 & 12,105 & 1.426 & 0.239 & 1.147 & 1.323 & 1.148 & 1.323 \\
\hline & 0.2 & 11,594 & 1.366 & 0.236 & 1.196 & 1.290 & 1.200 & 1.380 \\
\hline \multirow[t]{6}{*}{12} & SO & 8480 & - & 0.180 & 2.286 & 1.794 & 2.633 & 1.813 \\
\hline & 0 & 28,335 & 3.341 & 1.203 & 1.000 & 1.000 & 1.000 & 1.103 \\
\hline & 0.05 & 25,270 & 2.980 & 1.203 & 1.049 & 1.068 & 1.049 & 1.110 \\
\hline & 0.1 & 15,239 & 1.797 & 0.450 & 1.089 & 1.018 & 1.099 & 1.131 \\
\hline & 0.15 & 13,025 & 1.536 & 0.373 & 1.147 & 1.114 & 1.148 & 1.199 \\
\hline & 0.2 & 11,630 & 1.371 & 0.236 & 1.197 & 1.290 & 1.197 & 1.380 \\
\hline \multicolumn{9}{|c|}{ Istanbul European } \\
\hline \multirow[t]{5}{*}{17} & SO & 94,529 & - & 0.940 & 18.000 & 1.717 & 63.000 & 2.516 \\
\hline & 0 & $1,063,162$ & 11.247 & 14.546 & 1.000 & 1.000 & 1.000 & 1.000 \\
\hline & 0.05 & $1,055,691$ & 11.168 & 14.546 & 1.000 & 1.000 & 1.039 & 1.097 \\
\hline & 0.1 & $1,055,381$ & 11.165 & 14.546 & 1.056 & 1.229 & 1.075 & 1.229 \\
\hline & 0.15 & 433,460 & 4.585 & 7.382 & 1.116 & 1.229 & 1.145 & 1.229 \\
\hline \multirow[t]{5}{*}{22} & SO & 91,516 & - & 0.918 & 18.000 & 1.655 & 18.000 & 2.764 \\
\hline & 0 & $1,062,640$ & 11.612 & 14.546 & 1.000 & 1.000 & 1.000 & 1.000 \\
\hline & 0.05 & $1,055,124$ & 11.529 & 14.546 & 1.000 & 1.000 & 1.039 & 1.095 \\
\hline & 0.1 & $1,054,913$ & 11.527 & 14.546 & 1.000 & 1.000 & 1.075 & 1.046 \\
\hline & 0.15 & 432,234 & 4.723 & 7.382 & 1.116 & 1.057 & 1.145 & 1.062 \\
\hline \multicolumn{9}{|c|}{ Istanbul Anatolian } \\
\hline \multirow[t]{6}{*}{7} & SO & 13,805 & - & 0.491 & 3.531 & 2.242 & 4.000 & 2.242 \\
\hline & 0 & 58,731 & 4.254 & 1.314 & 1.000 & 1.000 & 1.000 & 1.000 \\
\hline & 0.05 & 58,295 & 4.223 & 1.314 & 1.041 & 1.039 & 1.041 & 1.039 \\
\hline & 0.1 & 55,404 & 4.013 & 1.314 & 1.041 & 1.039 & 1.061 & 1.062 \\
\hline & 0.15 & 47,506 & 3.441 & 0.704 & 1.147 & 1.085 & 1.147 & 1.085 \\
\hline & 0.2 & 26,460 & 1.917 & 0.401 & 1.191 & 1.146 & 1.194 & 1.146 \\
\hline \multirow[t]{6}{*}{10} & SO & 11,788 & - & 0.355 & 4.778 & 2.104 & 4.778 & 2.104 \\
\hline & 0 & 57,446 & 4.873 & 1.314 & 1.000 & 1.000 & 1.000 & 1.000 \\
\hline & 0.05 & 57,206 & 4.853 & 1.314 & 1.041 & 1.022 & 1.041 & 1.022 \\
\hline & 0.1 & 54,254 & 4.602 & 1.314 & 1.053 & 1.053 & 1.061 & 1.053 \\
\hline & 0.15 & 47,295 & 4.012 & 0.704 & 1.147 & 1.106 & 1.147 & 1.106 \\
\hline & 0.2 & 25,992 & 2.205 & 0.401 & 1.191 & 1.098 & 1.194 & 1.098 \\
\hline
\end{tabular}

$$
N U S=\max _{r \in O} \max _{\pi \in \cup_{S \in F^{*}} P_{r s}^{X}: v_{\pi}^{*}>0} \frac{d^{\pi}}{d_{r}^{*}},
$$

where $d_{r}^{*}=\min _{s \in F^{*}} d_{r s}^{*}$.

Loaded unfairness with respect to routes: Ratio of the experienced travel time of an evacuee to the experienced travel time of the fastest evacuee for the same origin-destination pair, where experienced travel time is the travel time measured in terms of the current congestion level:

$$
L U R=\max _{r \in O, s \in F^{*}} \max _{\pi \in P_{r s}^{*}: v_{\pi}^{*}>0} \frac{t^{\pi}}{t_{r s}^{*}},
$$

where $t^{\pi}$ is the congested travel time on route $\pi$ and $t_{r s}^{*}$ is the shortest congested travel time from origin $r$ to destination $s$.

Loaded unfairness with respect to shelters: Ratio of the experienced travel time of an evacuee to a shelter site, to the experienced travel time of an evacuee that is assigned to the most quickly reached shelter site for the same origin:

$$
L U S=\max _{r \in O} \max _{\pi \in \cup_{S \in F^{*}} P_{r s}^{x}: v_{\pi}^{*}>0} \frac{t^{\pi}}{t_{r}^{*}},
$$

where $t_{r}^{*}=\min _{s \in F^{*}} t_{r s}^{*}$.

Another measure we use is the maximum latency. The latency of a path $\pi$ is the experienced travel time on path $\pi$ and maximum latency is

$$
M L=\max _{r \in O, s \in F^{*}} \max _{\pi \in P_{r s}^{i}: v_{\pi}^{*}>0} t^{\pi} .
$$

Note that since we assume that all the evacuees enter the network at the same time, the network clearance time is equal to the maximum latency.

In Tables 4 and 5, for each instance, we report the total evacuation time, price of fairness, maximum latency, normal and loaded unfairness with respect to routes and shelters. We observe that when the demand is high compared to the capacity of 

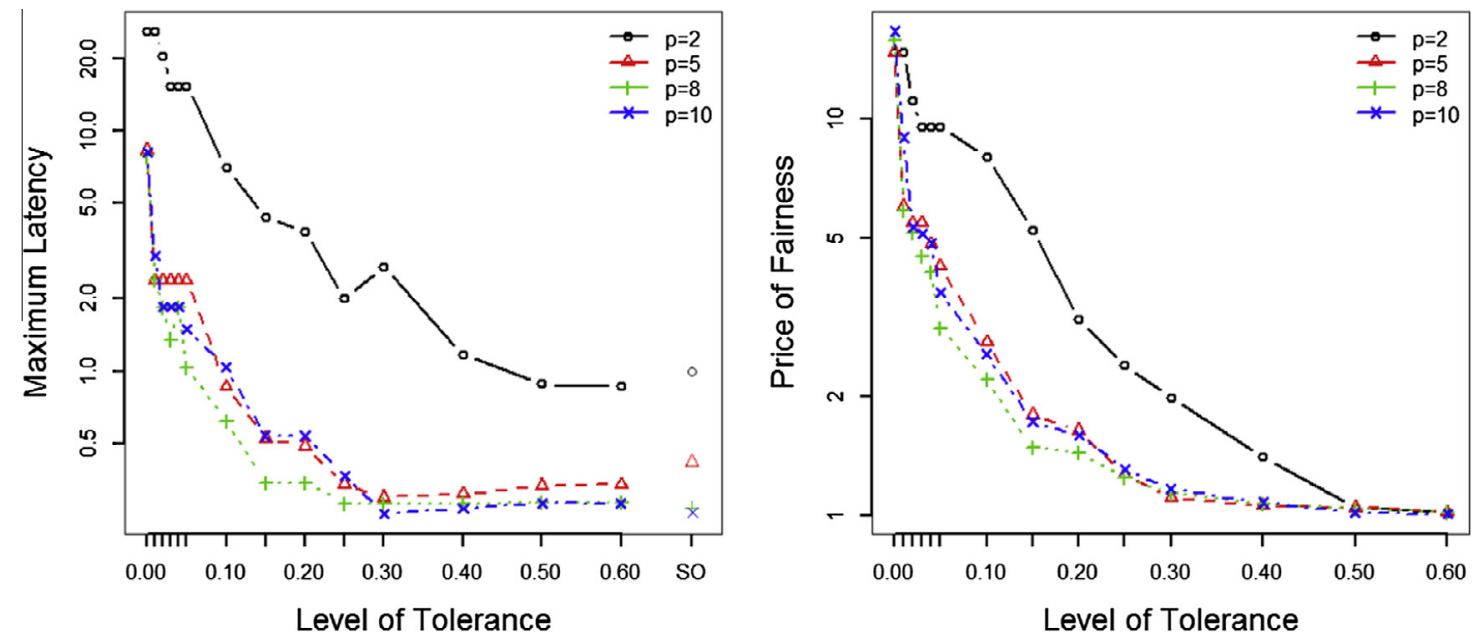

Fig. 4. The effect of level of tolerance on maximum latency and the tradeoff between level of tolerance and price of fairness, P-median1.

the network, as is the case for Sioux Falls 1 and Istanbul European, the price of fairness is very high. Even though this improves with increasing levels of tolerance, the difference between the performances of SO and CSO solutions is still significant. On the other hand, for Istanbul European with $p=17$, the normal unfairness of the SO solution with respect to routes and shelters is 18 and 63, respectively. In other words, there exist evacuees who are assigned to routes that are 63 times longer than the shortest route to the closest shelter. Hence the SO solution, even though very efficient compared to CSO solutions, is difficult to execute in practice. For the other networks, when $\lambda=0.2$, we obtain solutions that are almost as efficient as the SO solution and that are fair to evacuees. For instance, for the P-median1 network with $p=5$, the NUR and NUS for SO are 5.5 and 11.125, respectively, whereas they are 1.184 and 1.2 for the CSO solution with $\lambda=0.2$. The price of fairness for this tolerance level is 1.641 . The last evacuee to reach safety takes $0.487 \mathrm{~h}$, which is $0.417 \mathrm{~h}$ in the SO solution. For P-median6, the performance of SO and CSO solutions are very close in terms of total evacuation time and maximum latency, whereas the SO solutions are unfair, assigning some evacuees to shelters that are four times more distant compared to the closest ones. We also observe that the NA traffic assignment $(\lambda=0)$ is fair but may result in significant increase in the total evacuation time and the maximum latency. For P-median1, the price of fairness for these solutions are about 15 and the maximum latency may be as high as 20-30 times the one of the SO solution.

Fig. 4 illustrates the effect of level of tolerance on the maximum latency and the tradeoff between price of fairness and level of tolerance for the P-median1 network. As we increase the level of tolerance, the maximum latency tends to decrease for every $p$ value. When $p$ is two and the level of tolerance is zero, the maximum latency a vehicle incurs is more than $25 \mathrm{~h}$. When we open eight shelter sites and convince evacuees for a level of tolerance of 0.15 , maximum latency drops to 20 min. Since the traffic is distributed more evenly across the network as we increase the level of tolerance, the price of fairness tends to decrease for every value of $p$. Overall, we observe that having even some small tolerance has a positive impact on performance measures when $p$ is not very small. Fig. 5 depicts the same results for Istanbul Anatolian. In this case, small tolerance
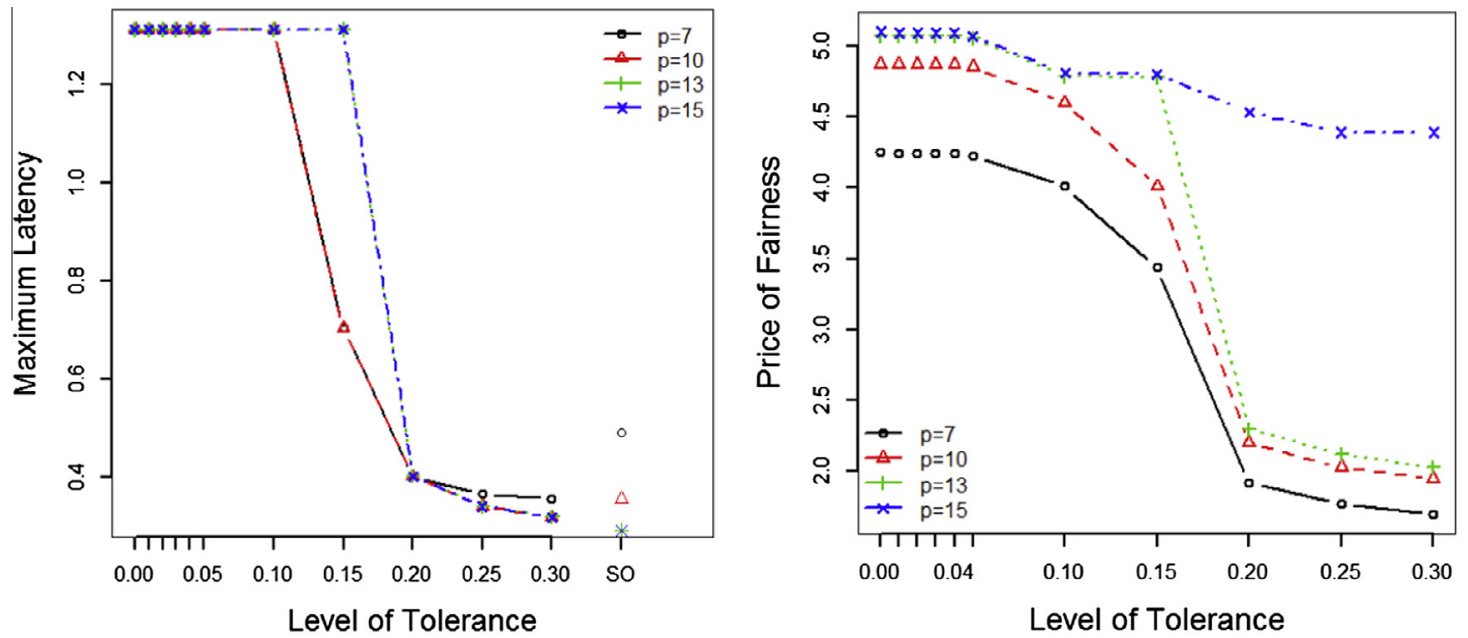

Fig. 5. The effect of level of tolerance on maximum latency and the tradeoff between level of tolerance and price of fairness, Istanbul Anatolian. 

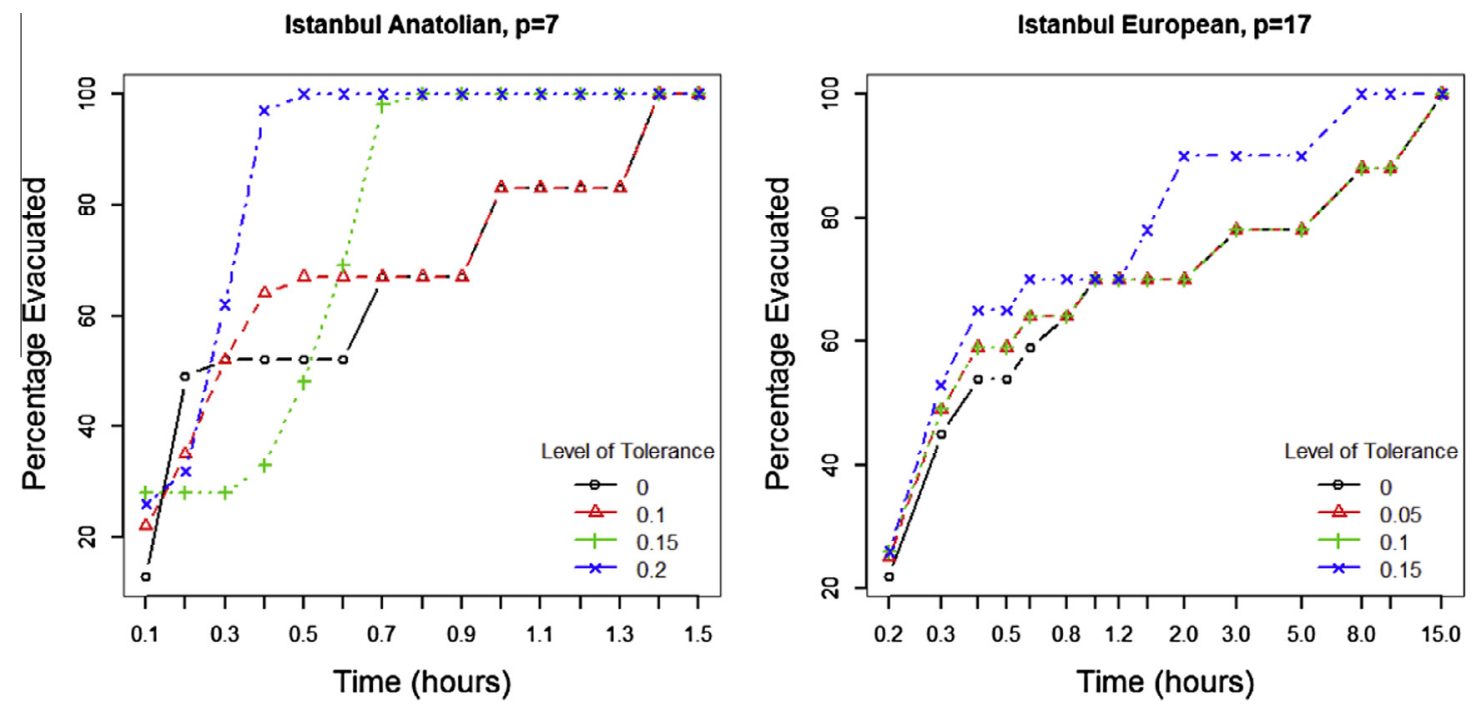

Fig. 6. The effect of the level of tolerance on percentage evacuated by a given time, Istanbul.

does not improve the performance measures. However, as $\lambda$ increases to 0.2 , the efficiency of the solution improves significantly.

Fig. 6 illustrates the effect of tolerance level on the percentage of demand that can be evacuated up to a specific time $T$. With the level of tolerance $\lambda=0$ and $p=17$ for the Istanbul European instance, it takes almost $8 \mathrm{~h}$ to evacuate $88 \%$ of the people in danger. When we increase $\lambda$ to only 0.15 the percentage of people evacuated in $2 \mathrm{~h}$ is $90 \%$. The case with Istanbul Anatolian instance is similarly striking. When $\lambda=0$ and $p=7$, it takes $1 \mathrm{~h}$ and $18 \mathrm{~min}$ to clear as much as $83 \%$ of people from the danger zone. If we can convince the evacuees for a level of tolerance of 0.2 , every evacuee reaches safety in $30 \mathrm{~min}$.

When we closely examine the Istanbul European instance we see that the demand at two origin nodes are very close to two shelter sites each and with a level of tolerance of 0.1 there are no other shelter sites at their proximity nor alternative paths within that limit. So each of these two nodes is assigned to a single shelter through a single path and since the amount of demand at these origin nodes is relatively large ( $21 \%$ of total evacuation demand), this assignment causes a congestion on these paths causing a latency of more than 7 and $14 \mathrm{~h}$, respectively. With an increase of level of tolerance to 0.15 , the demand at one of these two origins, specifically the one causing a latency of $14 \mathrm{~h}$, is distributed almost evenly to two shelter sites decreasing the congestion to a level that causes a latency of only an hour and a half, which in turn contributes by $12 \%$ to the $20 \%$ increase in the amount of people evacuated within $2 \mathrm{~h}$.

Finally, Fig. 7 illustrates the normal unfairness distributions with respect to paths and shelters for Istanbul Anatolian instance. Here, we depict the ratio of the population whose unfairness is at most a certain level. For instance, $89 \%$ of all
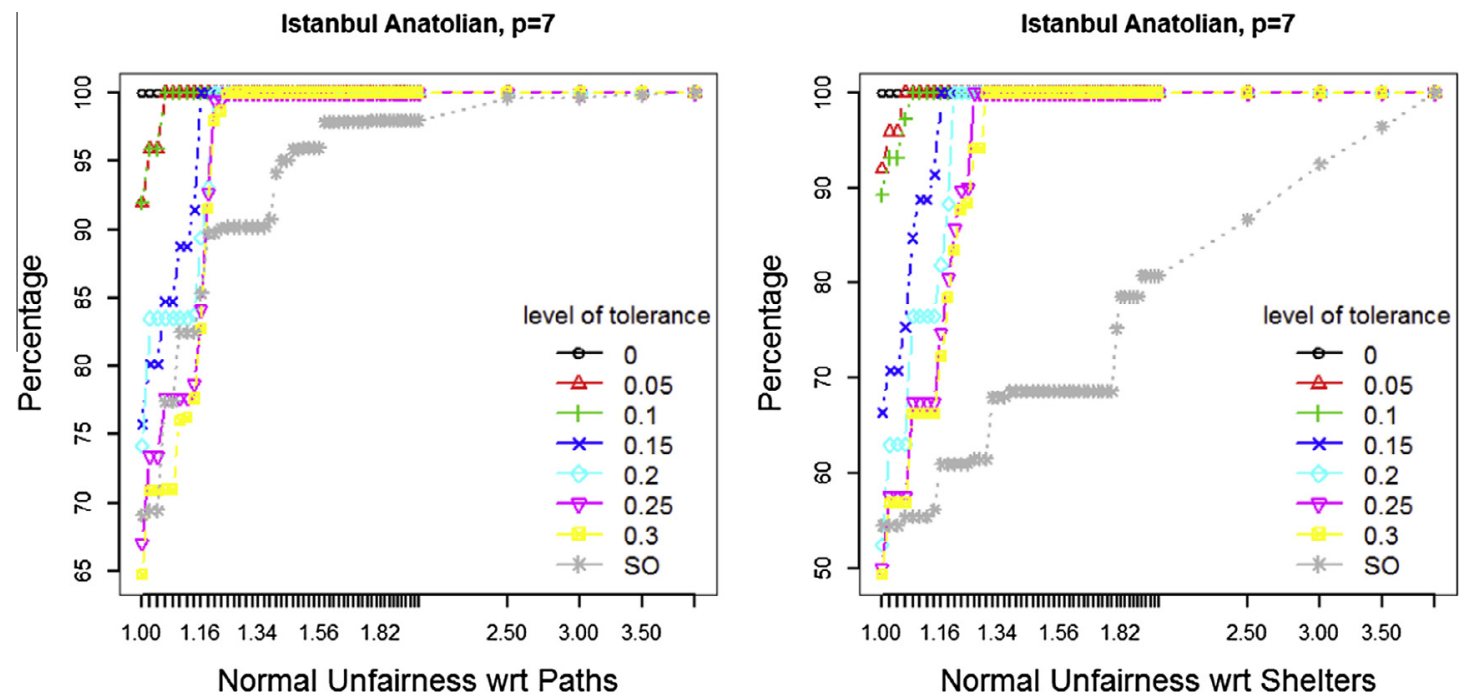

Fig. 7. Normal unfairness distributions with respect to paths and shelters for various tolerance levels, Istanbul Anatolian. 
Table 6

Comparison of SO, CSO and UE solutions for Istanbul Anatolian network.

\begin{tabular}{|c|c|c|c|c|c|c|c|}
\hline$p$ & model & Total evacuation time & ML & NUR & LUR & NUS & LUS \\
\hline \multirow[t]{3}{*}{7} & SO & 13,805 & 0.491 & 3.531 & 2.242 & 4.000 & 2.242 \\
\hline & $\operatorname{csO} \lambda=0.3$ & 23,423 & 0.355 & 1.259 & 1.116 & 1.3 & 1.141 \\
\hline & UE & 16,749 & 0.355 & 1.471 & 1.001 & 3.403 & 1.001 \\
\hline \multirow[t]{3}{*}{10} & SO & 11,788 & 0.355 & 4.778 & 2.104 & 4.778 & 2.104 \\
\hline & $\operatorname{CSO} \lambda=0.3$ & 22,956 & 0.317 & 1.259 & 1.116 & 1.3 & 1.141 \\
\hline & UE & 14,356 & 0.355 & 1.381 & 1.000 & 3.403 & 1.001 \\
\hline
\end{tabular}

evacuees for Istanbul Anatolian instance incur a normal unfairness of 1.16 with respect to paths when we employ a tolerance level of 0.2 with $p=7$. To rephrase, the route length of just $11 \%$ of evacuees is at least $16 \%$ more than that of the shortest path for their demand node - shelter site pairs. One can observe that there is substantial improvement in the unfairness distribution compared to the SO approach when $\lambda=0.3$. The improvement gets larger as $\lambda$ gets smaller.

To conclude, we compare our results with the case where evacuees would have perfect information and make their choices optimally in a UE setting. We use the Istanbul Anatolian instance for this purpose. We obtain results that are comparable with those of UE when $\lambda=0.3$. The results are reported in Table 6 .

When $\lambda=0.3$ and $p=7$ the performance of CSO is 69.7 percent worse than that of SO. The performance of UE is only 21.3 percent worse than that of SO for the same $p$. For the same instance, the time when the last evacuee leaves the network in CSO is the same as the one for UE but CSO performs better when $p=10$. The normal unfairness ratio with respect to shelters for UE when $p=7$ is more than 3.4 which is very close to the value of 4 for SO, i.e., there are evacuees who are assigned to routes that are 3.4 times longer than the shortest route to the closest shelter, which may be an unacceptable result for the evacuees without any knowledge of traffic conditions on the evacuation road network. The value for this ratio for CSO is 1.3. As expected the UE solution is very good for the loaded unfairness ratios since an equilibrium state prevails. But the values for these ratios for CSO are close to those of UE and are much better than those of the SO. We also compared the percentage of demand that can be evacuated up to a specific time $T$ for various levels of tolerance for CSO model and for UE model when $p=7$. UE evacuates $99.6 \%$ of the demand in $18 \mathrm{~min}$ whereas CSO evacuates $87 \%$ of the demand within the same time. Both models evacuate everyone to safety within 24 min.

\subsection{Capacitated shelters}

In our CSO model, we assume that the shelters have unlimited capacity and that their capacity can be planned according to the allocated demands. In this section, we analyze how fixed capacities affect the evacuation times and other performance measures.

To add capacity constraints to the CSO model, we omit the constraint $\sum_{s \in F} y_{s}=p$ and add the capacity constraints $\sum_{r \in O} \sum_{\pi \in P_{r s}^{i}} w_{r} v_{\pi} \leqslant K_{s} y_{s}, \forall s \in F$ where $K_{s}$ is the capacity of shelter $s$. We refer to the resulting model as "constrained system optimal model with capacitated shelters" and abbreviate it with CSO-CS. Likewise, we modified the SO model by omitting the constraint $\sum_{s \in F} y_{s}=p$ and adding the capacity constraints $0 \leqslant f_{s} \leqslant K_{s} y_{s}, \forall s \in F$. The resulting model is called SO-CS.

We use the Istanbul European and Istanbul Anatolian networks in this experiment. We take the shelter capacities from Kırıkçı (2012). With the original capacities, there is no feasible solution for the Istanbul European network for all the $\lambda$ values up to 0.2 . For that reason, we use two times the original capacities for that network. With these updated shelter capacities we find optimal solutions when $\lambda=0.15$ and $\lambda=0.2$. For the Istanbul Anatolian network, the problem is feasible for $\lambda \geqslant 0.05$.

In Table 7, we report the results for Istanbul European and Istanbul Anatolian networks for various levels of tolerance. For each instance, we report the number of shelters opened (\#shelters), total evacuation time, price of fairness, maximum

Table 7

Efficiency and fairness with capacitated shelters.

\begin{tabular}{|c|c|c|c|c|c|c|c|c|}
\hline$\lambda$ & \# shelters & Total evacuation time & $\rho(\lambda)$ & ML & NUR & LUR & NUS & LUS \\
\hline \multicolumn{9}{|l|}{ Istanbul European } \\
\hline SO & 28 & 90,426 & - & 0.943 & 18 & 1.658 & 30.5 & 2.883 \\
\hline 0.15 & 23 & 473,085 & 5.232 & 7.382 & 1.116 & 1.057 & 1.145 & 5.329 \\
\hline 0.2 & 22 & 463,249 & 5.123 & 7.382 & 1.116 & 1.057 & 1.156 & 5.329 \\
\hline \multicolumn{9}{|l|}{ Istanbul Anatolian } \\
\hline SO & 14 & 11,755 & - & 0.338 & 4.778 & 2.601 & 15.444 & 8.225 \\
\hline 0.05 & 8 & 207,162 & 17.624 & 2.835 & 1.049 & 1.004 & 1.050 & 1.032 \\
\hline 0.1 & 11 & 54,795 & 4.662 & 1.314 & 1.008 & 1.006 & 1.061 & 1.265 \\
\hline 0.15 & 11 & 54,795 & 4.662 & 1.314 & 1.058 & 1.058 & 1.146 & 1.265 \\
\hline 0.2 & 10 & 39,855 & 3.391 & 0.935 & 1.191 & 1.044 & 1.194 & 4.244 \\
\hline 0.25 & 10 & 38,333 & 3.261 & 0.939 & 1.191 & 1.067 & 1.247 & 4.261 \\
\hline 0.3 & 10 & 38,320 & 3.260 & 0.939 & 1.259 & 1.080 & 1.288 & 4.258 \\
\hline
\end{tabular}




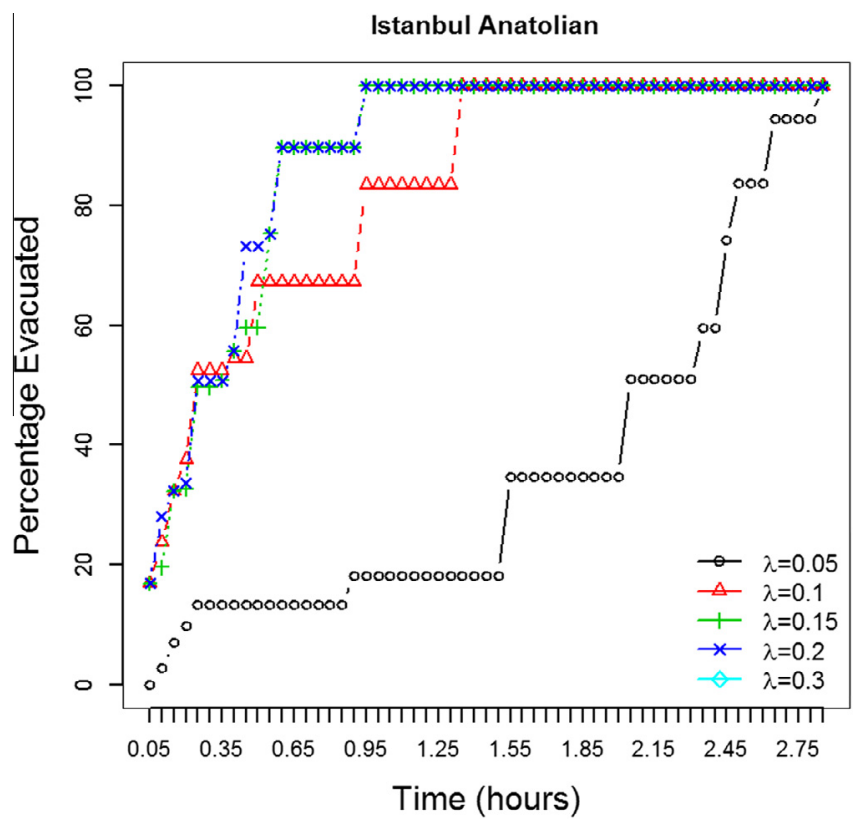

Fig. 8. The effect of the level of tolerance on percentage evacuated by a given time with capacitated shelters, Istanbul Anatolian.

latency, normal and loaded unfairness with respect to routes and shelters. Clearly, the effect of tolerance on the total evacuation time is much higher when the shelters are capacitated. Having no tolerance results in infeasibility for both networks. For the Anatolian network, increasing the tolerance level from 0.05 to 0.1 decreases the total evacuation time to its quarter and decreases the maximum latency to its half. The maximum latency values for SO-CS model are similar compared to the uncapacitated case for both networks, but they tend to increase for CSO-CS model. The normal unfairness ratio with respect to shelters for SO-CS model for Istanbul European network increases drastically. For the uncapacitated case the worst value over any $p$ for normal unfairness ratio with respect to shelters is 18 and for the capacitated case this number is 30.5 . With the SO model, the loaded unfairness ratio with respect to shelters for the Istanbul Anatolian network shows a large increase compared to the uncapacitated case, assigning evacuees to shelters more than 8 times longer in travel time compared to the shelter with the shortest travel time. For the CSO model there is a significant increase in the loaded unfairness ratio with respect to shelters for large values of $\lambda$, but the model still performs much better than SO model in this respect.

Fig. 8 illustrates the percentage of demand that can be evacuated up to a specific time $T$ for various levels of tolerance for CSO model with capacitated shelters for the Istanbul Anatolian network. In the uncapacitated case, when $\lambda=0.2$ everyone reaches safety within $30 \mathrm{~min}$, for the capacitated case the time to evacuate everyone is almost one hour.

Overall, for our networks, we can conclude that in the presence of capacity restrictions, it is crucial to have some tolerance for a better system performance.

\section{Conclusions}

When planning for an evacuation the desirable goal is to minimize the total evacuation time by considering a SO approach. Since this approach may guide evacuees to paths that are longer than the ones they would take, its solution may be inapplicable. On the other hand, since disasters are rare events and it is not possible for evacuees to know the traffic conditions on the road network, they will rather tend to reach the nearest shelter by taking a shortest route (or shortest free flow time route). However, if evacuees know that they are being treated fairly among others and also that their relatively small sacrifice by taking a route within a tolerance level instead of the shortest route will contribute to them and to the overall benefit of the evacuation process in a great deal, they can consent to the CSO solution.

We propose a novel model that captures this human behavior by also taking into account the impact of location of shelter sites. It turns out that the decision of how many shelter sites to open and where to locate them is critical to the evacuation planners. For our instances, we observed that as we open more shelter sites and convince the evacuees for a higher level of tolerance, the total evacuation time and the maximum latency decrease and the percentage of people evacuated up to a specified time increases. This guarantees having a more efficient evacuation plan, compared to the case where evacuees act selfishly and take the shortest route to the nearest shelter site. However, one needs to be careful about opening more shelter sites, i.e., if the potential shelter sites are not chosen properly it may not be advantageous to open more shelters.

As the level of tolerance increases, so does unfairness, both in terms of paths and shelters. By considering the trade off between unfairness and price of fairness, a carefully chosen level of tolerance can be a balance between these two conflicting objectives. 
In our approach, we ignored uncertainty that is inherent in evacuation management. As a continuation of our study, we aim to extend the current work to incorporate uncertainty in the demand and the network using two stage stochastic programming where the shelter sites are decided in the first stage and routing decisions are taken in the second stage. An alternative approach may be to add chance constraints that guarantee that all the population is evacuated within a specified time with high probability.

Another interesting extension may be to add lower bounds on the number of evacuees allocated to open shelters so that the limited resources to operate and serve shelters are used efficiently.

Incorporating uncertainty or constraints on the number of evacuees allocated to shelters will certainly make the problem more difficult. In that case, it may be advantageous not to work with large relaxations that involve variables for all possible paths and to generate these variables when required within a column generation framework.

\section{Acknowledgement}

We thank the referees for their suggestions that improved the presentation and the content of the paper. This research is supported by TUBITAK, The Scientific and Technological Research Council of Turkey with project number 213M434. The research of the third author is supported by the Turkish Academy of Sciences.

\section{References}

Alçada-Almeida, L., Tralhão, L., Santos, L., Coutinho-Rodrigues, J., 2009. A multiobjective approach to locate emergency shelters and identify evacuation routes in urban areas. Geographical Analysis 41 (1), 9-29.

Alizadeh, F., Goldfarb, D., 2003. Second-order cone programming. Mathematical Programming 95 (1), 3-51.

Barrett, B., Ran, B., Pillai, R., 2000. Developing a dynamic traffic management modeling framework for hurricane evacuation. Transportation Research Record: Journal of the Transportation Research Board 1733 (2000), 115-121.

Beasley, J.E., 1990. Or-library: distributing test problems by electronic mail. Journal of the Operational Research Society, $1069-1072$.

Branston, D., 1976. Link capacity functions: A review. Transportation Research 10 (4), 223-236.

Byers, T.H., Waterman, M.S., 1984. Technical note, Determining all optimal and near-optimal solutions when solving shortest path problems by dynamic programming. Operations Research 32 (6), 1381-1384.

Chen, H.C., Friedman, J.W., Thisse, J.F., 1997. Boundedly rational nash equilibrium: a probabilistic choice approach. Games and Economic Behavior 18 (1), 32-54.

Chiu, Y.C., Zheng, H., Villalobos, J., Gautam, B., 2007. Modeling no-notice mass evacuation using a dynamic traffic flow optimization model. IIE Transactions 39 (1), 83-94.

CNN.com, 2001. CNN live at daybreak: Escaping a hurricane <http://edition.cnn.com/TRANSCRIPTS/>

Correa, J.R., Schulz, A.S., Stier-Moses, N.E., 2005. On the inefficiency of equilibria in congestion games. In: Integer Programming and Combinatorial Optimization. Springer, pp. 167-181.

Correa, J.R., Schulz, A.S., Stier-Moses, N.E., 2007. Fast, fair, and efficient flows in networks. Operations Research 55 (2), $215-225$.

Coutinho-Rodrigues, J., Tralhão, L., Alçada-Almeida, L., 2012. Solving a location-routing problem with a multiobjective approach: the design of urban evacuation plans. Journal of Transport Geography 22, 206-218.

Cova, T.J., Johnson, J.P., 2003. A network flow model for lane-based evacuation routing. Transportation Research Part A 37 (7), $579-604$.

EM-DAT, 2013. Natural disasters trends. The International Disaster Database, Centre for Research on the Epidemiology of Disasters (CRED) <http:// www.emdat.be/natural-disasters-trends>.

Erdik, Mustafa, Durukal, Eser, 2008. Earthquake risk and its mitigation in istanbul. Natural Hazards 44 (2), $181-197$.

Faturechi, Reza, Miller-Hooks, Elise, 2014. Travel time resilience of roadway networks under disaster. Transportation Research Part B 70, 47-64.

FEMA, Federal Emergency Management Agency, 2010. Developing and maintaining emergency operations plans: Comprehensive preparedness guide (cpg) 101, version $2.0<$ http://training.fema.gov/>.

Frank, M., Wolfe, P., 1956. An algorithm for quadratic programming. Naval Research Logistics Quarterly 3 (1-2), 95-110.

Galindo, G., Batta, R., 2013. Review of recent developments in OR/MS research in disaster operations management. European Journal of Operational Research.

Gürel, S., 2011. A conic quadratic formulation for a class of convex congestion functions in network flow problems. European Journal of Operational Research 211 (2), 252-262.

Hamacher, H.W., Heller, S., Rupp, B., 2011. Flow location (flowloc) problems: dynamic network flows and location models for evacuation planning. Annals of Operations Research, 1-20.

Hu, Zhi-Hua, Sheu, Jiuh-Biing, Xiao, Ling, 2014. Post-disaster evacuation and temporary resettlement considering panic and panic spread. Transportation Research Part B 69, 112-132.

IFRC, 2011. The red cross red crescent approach to disaster and crisis management position paper, Geneva. International Federation of Red Cross and Red Crescent Societies <http://www.ifrc.org/PageFiles/91314/1209600-DM-Position-Paper-EN.pdf>.

IMM-JICA, 2002. The Study on a Disaster Prevention/Mitigation Basic Plan in Istanbul Including Seismic Microzonation in the Republic of Turkey. Tech. rep. The Istanbul Metropolitan Municipality Japan International Cooperation Agency.

Jahn, O., Möhring, R.H., Schulz, A.S., 2000. Optimal routing of traffic flows with length restrictions in networks with congestion. In: Operations Research Proceedings 1999. Springer, pp. 437-442.

Jahn, O., Möhring, R.H., Schulz, A.S., Moses, N.E.S., 2003. System-optimal routing of traffic flows with user constraints in networks with congestion. MIT Sloan School of Management.

Jahn, O., Möhring, R.H., Schulz, A.S., Stier-Moses, N.E., 2005. System-optimal routing of traffic flows with user constraints in networks with congestion. Operations Research 53 (4), 600-616.

Kariv, O., Hakimi, S.L., 1979. An algorithmic approach to network location problems. ii: The p-medians. SIAM Journal on Applied Mathematics 37 (3), 539 560.

Kırıkçı, C., 2012. Determination of Shelter Locations and Evacuation Routes for a Possible Earthquake in the City of Istanbul (Master's thesis). Department of Industrial Engineering, Bilkent University.

Kongsomsaksakul, S., Yang, C., Chen, A., 2005. Shelter location-allocation model for flood evacuation planning. Journal of the Eastern Asia Society for Transportation Studies 6 (1), 4237-4252.

Koutsoupias, E., Papadimitriou, C., 1999. Worst-case equilibria. In: STACS 99. Springer, pp. 404-413.

Kulshrestha, A., Wu, D., Lou, Y., Yin, Y., 2011. Robust shelter locations for evacuation planning with demand uncertainty. Journal of Transportation Safety \& Security 3 (4), 272-288. 
Li, A.C.Y., Nozick, L., Xu, N., Davidson, R., 2012. Shelter location and transportation planning under hurricane conditions. Transportation Research Part E 48 (4), 715-729.

Li, Z., Zhao, X., 2008. Integrated-equilibrium Routing of Traffic Flows with Congestion, vol. 17. World Congress, pp. 16065-16070.

Lindell, M.K., Prater, C.S., 2007. Critical behavioral assumptions in evacuation time estimate analysis for private vehicles: Examples from hurricane research and planning. Journal of Urban Planning and Development 133 (1), 18-29.

Litman, Todd, 2006. Lessons from katrina and rita: What major disasters can teach transportation planners. Journal of Transportation Engineering 132 (1), $11-18$.

Liu, Simin, Quenemoen, Lynn E, Malilay, Josephine, Noji, Eric, Sinks, Thomas, Mendlein, James, 1996. Assessment of a severe-weather warning system and disaster preparedness, calhoun county, alabama, 1994. American Journal of Public Health 86 (1), $87-89$.

Lou, Y., Yin, Y., Lawphongpanich, S., 2010. Robust congestion pricing under boundedly rational user equilibrium. Transportation Research Part B 44 (1), 1528.

Mahmassani, H.S., Chang, G.L., 1987. On boundedly rational user equilibrium in transportation systems. Transportation Science 21 (2), 89-99.

Mahmassani, H.S., Liu, Y.H., 1999. Dynamics of commuting decision behaviour under advanced traveller information systems. Transportation Research Part C 7 (2), 91-107.

Nemirovski, A., Tal, A.B., 2001. Lectures on modern convex optimization: Analysis, algorithms, and engineering applications. MPS-SIAM Series on Optimization.

Ng, M., Park, J., Waller, S.T., 2010. A hybrid bilevel model for the optimal shelter assignment in emergency evacuations. Computer-Aided Civil and Infrastructure Engineering 25 (8), 547-556.

Olsthoorn, F., 2012. The Price of Anarchy (Master's thesis). Mathematisch Instituut, Universiteit Leiden.

OR-Library. 1990. P-median instances <http://people.brunel.ac.uk/mastjjb/jeb/info.html>.

Pel, A.J., Bliemer, M.C.J., Hoogendoorn, S.P., 2012. A review on travel behaviour modelling in dynamic traffic simulation models for evacuations, Transportation 39 (1), 97-123.

Roughgarden, T., 2002. Selfish Routing (Ph.D. thesis). Cornell University.

Schulz, A.S., Moses, N.S., 2003. On the performance of user equilibria in traffic networks. In: Proceedings of the Fourteenth Annual ACM-SIAM Symposium on Discrete Algorithms. Society for Industrial and Applied Mathematics, pp. 86-87.

Schulz, A.S., Stier-Moses, N.E., 2006. Efficiency and fairness of system-optimal routing with user constraints. Networks 48 (4), $223-234$.

Shen, Z.J.M., Pannala, J., Rai, R., Tsoi, T.S., 2008. Modeling Transportation Networks During Disruptions and Emergency Evacuations. University of California Transportation Center.

Sherali, H.D., Carter, T.B., Hobeika, A.G., 1991. A location-allocation model and algorithm for evacuation planning under hurricane/flood conditions. Transportation Research Part B 25 (6), 439-452.

Sheu, Jiuh-Biing, Pan, Cheng, 2014. A method for designing centralized emergency supply network to respond to large-scale natural disasters. Transportation Research Part B 67, 284-305.

Simon, H.A., 1955. A behavioral model of rational choice. The Quarterly Journal of Economics 69 (1), 99-118.

Southworth, F., 1991. Regional Evacuation Modeling: A State-of-the-art Review. Tech. rep., Oak Ridge National Laboratory Oak Ridge, TN, USA

Szeto, W.Y., Lo, H.K., 2006. Dynamic traffic assignment: properties and extensions. Transportmetrica 2 (1), 31-52.

TAM, 1964. Traffic Assignment Manual. Bureau of Public Roads, U.S. Department of Commerce.

TNTP, 2001. Transportation Network Test Problems <http://www.bgu.ac.il/bargera/tntp/>.

TRB, 2008. Committee on the Role of Public Transportation in Emergency Evacuation, Transportation Research Board of the National Academies. The role of transit in emergency evacuation Special Report(294). <http://www.TRB.org/>.

TÜIK, 2013. Statistics Institute of Turkey, Adrese dayali nufus kayit sistemi <http://www.tuik.gov.tr/>.

Yamada, T., 1996. A network flow approach to a city emergency evacuation planning. International Journal of Systems Science 27 (10), 931-936.

Yazıcı, M.A., 2010. Introducing Uncertainty into Evacuation Modeling via Dynamic Traffic Assignment with Probabilistic Demand and Capacity Constraints (Ph.D. thesis). Rutgers University-Graduate School-New Brunswick.

Yazıcı, M.A., Özbay, K., 2007. Impact of probabilistic road capacity constraints on the spatial distribution of hurricane evacuation shelter capacities. Transportation Research Record: Journal of the Transportation Research Board 2022 (1), 55-62.

Zhou, B., Li, X., 2012. User equilibrium with length constrained users. In: Computational Sciences and Optimization (CSO), 2012 Fifth International Joint Conference on. IEEE, pp. 494-499. 